\title{
A perturbative framework for jet quenching
}

\author{
Korinna C. Zapp, ${ }^{a, b}$ Frank Krauss $^{b}$ and Urs A. Wiedemann ${ }^{a}$ \\ ${ }^{a}$ Department of Physics, CERN, Theory Unit, \\ CH-1211 Geneva 23, Switzerland \\ ${ }^{b}$ Institute for Particle Physics Phenomenology, Durham University, \\ Durham DH1 3LE, U.K. \\ E-mail: korinna.zapp@cern.ch, frank.krauss@durham.ac.uk, \\ Urs.Wiedemann@cern.ch
}

ABSTRACT: We present a conceptually new framework for describing jet evolution in the dense medium produced in ultra-relativistic nucleus-nucleus collisions using perturbative QCD and its implementation into the Monte Carlo event generator JEwEL. The rescattering of hard partons in the medium is modelled by infrared continued pQCD matrix elements supplemented with parton showers. The latter approximate higher order realemission matrix elements and thus generate medium-induced gluon emissions. The interplay between different emissions is governed by their formation times. The destructive interference between subsequent scattering processes, the non-Abelian version of the Landau-Pomeranchuk-Migdal effect, is also taken into account. In this way the complete radiation pattern is consistently treated in a uniform way. Results obtained within this minimal and theoretically well constrained framework are compared with a variety of experimental data susceptible to jet-quenching effects at both RHIC and the LHC. Overall, a good agreement between data and simulation is found. This new framework also allows to identify and quantify the dominant uncertainties in the simulation, and we show some relevant examples for this.

KEYwords: Heavy Ion Phenomenology 


\section{Contents}

1 Introduction 1

2 Parton shower in a medium 5

2.1 Reminder: parton showers 5

$\begin{array}{lll}2.2 & \text { Scattering in a medium and parton showers } & 7\end{array}$

2.3 Reminder: probabilistic interpretation of the LPM effect 9

3 Details of the implementation $\quad 10$

$\begin{array}{ll}3.1 \text { Event generation } & 10\end{array}$

$\begin{array}{lll}3.2 & \text { In-medium matrix elements and their infrared continuation } & 10\end{array}$

$\begin{array}{lll}3.3 & \text { Parton shower } & 11\end{array}$

$\begin{array}{lll}3.4 & \text { Time evolution } & 12\end{array}$

3.5 Implementation of the LPM effect in JEWEL 12

$\begin{array}{lll}3.6 & \text { Recoils, colour flows, and hadronisation } & 14\end{array}$

$\begin{array}{lll}3.7 & \text { Medium model } & 14\end{array}$

4 Results $\quad \mathbf{1 5}$

$\begin{array}{lll}4.1 & \text { Validation } & 15\end{array}$

$\begin{array}{lll}4.2 & \text { Hadron suppression } & 19\end{array}$

$\begin{array}{lll}4.3 & \text { Modification of jets } & 21\end{array}$

4.4 Uncertainties 26

$\begin{array}{llr}5 & \text { Conclusions } & 27\end{array}$

\section{Introduction}

High momentum transfer processes occur abundantly in hadronic interactions at collider energies. This is not only true for proton-proton collisions at the LHC and other similar collider experiments before it, but also for nucleus-nucleus collisions at RHIC and at the LHC. Such processes are well understood and calculable in perturbative QCD and they can be simulated with modern Monte Carlo event generators [1-3] using QCD matrix elements supplemented with parton showers [4]. The latter resum the dominant collinear logarithms associated to the emission of additional partons to leading (and partly sub-leading) logarithmic accuracy, with many improvements and reformulations in the past decades [5-13]. In the present work, we discuss how such techniques can be used to formulate a general framework for the parton shower evolution in the presence of dense QCD matter produced in nucleus-nucleus collisions.

Simple considerations, based on scale arguments and the uncertainty relation, indicate that final state parton showers evolve for up to several $\mathrm{fm} / \mathrm{c}$, which is comparable to the 
transverse size of the dense QCD matter produced in the spatially extended overlap region of ultra-relativistic nucleus-nucleus collisions. Therefore it can be expected that the QCD parton showers introduced and studied in the vacuum will be subjected to medium modifications in nucleus-nucleus collisions. Data from RHIC and the LHC strongly support this view. In particular, in comparison to standard perturbative benchmarks from $p p$-collisions, single inclusive hadron spectra at high transverse momentum are strongly suppressed, by up to a factor of 5 at RHIC [14, 15], and by up to a factor of 7 at the LHC [16-18]. Over the last two years, data on reconstructed jets in heavy-ion collisions have provided further, more differential information about such medium modifications of QCD radiation. In particular, measurements of the di-jet asymmetry $[19,20]$ and the inclusive jet suppression $[21,22]$ indicate that the interaction of parton showers with the surrounding QCD medium can dissipate a significant fraction of its total transverse energy to large angles, outside typical jet cones. ${ }^{1}$ On the other hand, the fragmentation functions inside the jet cone appear to experience only moderate medium modifications [25, 26].

The modification of leading fragments has been anticipated more than two decades ago [27-29] and triggered the development of a large number of jet quenching models in subsequent years. For recent reviews on such models and their comparison to data from RHIC and the LHC, see refs. [30-33]. In the following, we limit our discussion to highlighting the main similarities and the main differences between our proposal and some of these previous works. We start by recalling that there are essentially three qualitatively different analytically formulated approaches to medium-induced parton energy loss, all of which based on strong assumptions about kinematics and/or medium properties.

Firstly, starting with refs. [27, 28] there are analytical studies of collisional parton energy loss, in which highly energetic partons lose energy and randomise momentum in consecutive partonic $2 \rightarrow 2$ scatterings with partonic components of the medium. These studies do not assume particular kinematical constraints. They are strongly limited, however, in that they do not include medium-induced parton emission processes.

A complementary mechanism, based on analytical studies of radiative parton energy loss through modifications of the emission pattern of secondary partons, was proposed in refs. [34-39]. For technical reasons, the emissions in this approach have been limited to close-to-eikonal kinematics, neglecting recoil effects. Also, typically the emission of only one gluon from a single highly energetic parton is considered. Adjusted with a number of phenomenologically motivated requirements, the simple picture introduced in the publications above has been employed in model studies in the past years [40-43]. Recent analytical studies improve on some approximations by considering gluon emission from eikonal antennae $[44,45]$, and by providing a differential formulation of the colour flow in the multiple scattering process [46].

In addition, radiative parton energy loss calculations illustrated explicitly that by scattering on several spatially separated partons, the distribution of emitted gluons is modified by destructive interference effects. In the eikonal limit, these interference patterns

\footnotetext{
${ }^{1}$ Reconstructed jets in heavy ion collisions are by now typically identified through the anti- $k_{\perp}$ jet algorithm [23]. The jet catchment areas that the anti- $k_{\perp}$ algorithm associates to jets are approximately circular [24], and we shall refer to them for simplicity as 'cones' in the following.
} 
are understood in terms of the non-Abelian Landau-Pomeranchuk-Migdal (LPM) effect. This effect can be implemented in a probabilistic formulation based on formation time constraints, and lead to the first simulations taking this effect consistently into account [47].

By now, however, it is widely accepted that analytical studies of radiative parton energy loss provide only very limited constraints on phenomenological models implemented in simulation codes [48], mainly because they are restricted to a small part of the relevant kinematics and because they only insufficiently constrain how medium-effects modify the vacuum structure of the parton shower. There have been strong efforts in recent years to overcome these deficiencies in Monte Carlo formulations of medium-modified parton showers [49-56]. Many of these models use parton formation times to specify destructive interference in parton emissions and the spatio-temporal embedding of the parton shower in the medium. This is closely related to the procedure that we propose in the present work. On the other hand, the modelling of medium-induced gluon emissions typically makes use of analytical results obtained in the eikonal limit. Thus, although the Monte Carlo models can improve on aspects like implementing exact energy-momentum conservation, it should be stressed that they still suffer from the numerically large systematic uncertainties and conceptual limitations related to using the analytical results outside their strict region of validity. This is why we believe it is important not only to formulate models with tractable assumptions but also to supplement them with a well-defined way to quantify related uncertainties.

Finally, analytical studies of parton energy loss in strongly coupled non-Abelian plasmas with known gravity duals should be mentioned, for a recent review cf. [57]. This approach is qualitatively different from the one proposed here and we do not discuss it further in the present work.

In this paper, a conceptually new approach to jet quenching is formulated, which consistently includes both collisional and radiative parton energy loss mechanisms. It is based on known properties of perturbative QCD in non-eikonal kinematics and their continuation to the infrared region. This formulation consistently treats the scattering of an energetic parton off a medium constituent in perturbative QCD using standard techniques. The resulting interaction is described at leading order through standard $2 \rightarrow 2$ QCD matrix elements, which are valid in the full perturbative regime and can easily be extended into the non-perturbative region with some simple, predictive parametrisations. Further, following the standard description of all hard scattering events at colliders, large logarithms associated to collinear singularities occurring in matrix elements for subsequent real emissions are resummed by the parton shower and thus dealt with in a probabilistic language. This picture provides a well understood and systematically improvable approximation to extra gluon emissions.

In this way both collisional and radiative energy loss are generated consistently as part of a more general description of scattering processes in perturbative QCD. This approach includes in a natural way also radiation off the scattering centre and generates configurations with any number of additional partons. So-called flavour conversion processes that transfer essentially the entire energy of a parton to a different parton are also automatically included and the framework can easily accommodate heavy quark flavours. 
While this approach offers a general, flexible and largely well controlled framework for describing jet evolution in the presence of a dense, strongly interacting medium, it is not free of assumptions:

1. The medium as seen by the jet is a collection of partons with a certain distribution in phase space. For scattering processes with a very high scale (resolution) this is certainly a reasonable assumption. Hard processes happen on very short time scales, which are much shorter than any interaction among the partons forming the medium. For such processes, the partonic constituents of the medium are resolved by the jet, the scattering partner is thus a quasi-free parton. This essentially is the same argument that is employed, for example, in deeply virtual scattering and corresponding factorisation theorems. The actual assumption here is that this factorisation is valid for all the interactions of the jet in the medium. The actual phase space distribution of scattering partners is irrelevant for the qualitative picture underlying the formulation of jet-medium interactions, but of course quantitatively impact on the results.

2. By continuing the $p Q C D$ matrix elements into the infra-red region the dominant effect of soft scatterings can be included. Since it is impossible to restrict the framework in a meaningful way to include perturbatively hard scatterings in the medium only, some modelling of soft scatterings is unavoidable. While clearly other approaches are conceivable and may be equally legitimate, a suitable infra-red continued version of perturbation theory could be considered as a minimal model, in which the exact form of the infra-red continuation and the exact choice of the regulator are a source of uncertainties. These uncertainties, however, can be studied systematically and, with sufficient data, most likely be further constrained. It should be noted, though, that in particular the total scattering cross section in the medium depends on these choices.

3. The interplay between different sources of radiation is governed by the formation times of the emissions. As it is clearly unphysical to assume that additional emissions happen instantaneously, there will be situations where several radiation processes occur at the same time. In the framework presented here, each emission is ascribed a formation time and for two competing emissions the one with the shorter formation time is accepted, while the other will not be realised. However plausible this seems, this nevertheless goes beyond standard parton showers, which have no notion of space-time evolution. Furthermore, there are only parametric estimates of the formation time. The resulting freedom of an exact choice will yield different admixtures of different processes in the same situations. This, again, gives rise to some uncertainties, which, however, are easily quantifiable.

4. The physical picture behind the LPM-effect derived from results in the eikonal limit is valid also in non-eikonal kinematics. In calculations carried out in the eikonal limit it was found that there is a destructive interference between gluon emissions induced by subsequent scattering processes when the distance between the scattering centres is shorter than the gluon formation time. This is the non-Abelian analogue 
of the Landau-Pomeranchuk-Migdal (LPM) effect. A probabilistic interpretation of this phenomenon based on a physical picture was derived and validated in the eikonal limit. The validity of this picture also in non-eikonal kinematics is only assumed. A straightforward generalisation of the algorithm can be used to include the LPM-effect in the framework presented here.

In the remainder of this publication, the construction paradigms above will be further worked out. To put the different ingredients into perspective, to further develop the language employed, and to define our notation, however, the reader is first reminded of a few concepts that will be used throughout, cf. section 2. Details of the implementation relevant for a better understanding of our model are presented in section 3. In section 4 we confront this new model with a broad range of data, starting from those vacuum observables in $e^{-} e^{+}$and $p p$ collisions, which fix the parton shower and hadronisation parameters and thus leaves us with a minimal set of relevant parameters. By comparing to a number of data from RHIC and LHC, which show jet quenching effects, we highlight the versatility of our model. We also make a non-trivial, testable prediction there. Finally, we summarise with some concluding remarks in section 5 .

\section{Parton shower in a medium}

In this section, the ingredients necessary to construct an in-medium parton shower following the paradigms outlined above will be discussed. In section 2.1 the parton shower picture in the vacuum is briefly summarised. The rescattering of a hard parton in the medium is discussed in section 2.2, and in section 2.3 the probabilistic interpretation of the LPM-effect in the eikonal limit is recapitulated. Details of its generalisation to the full phase space are given in section 3.5 .

\subsection{Reminder: parton showers}

In the collinear limit, QCD real emission matrix elements and their phase space factorise such that the differential cross section for any process with an additional parton in the final state, $\mathrm{d} \sigma_{n+1}$, can be expressed by the cross section for the production of an $n$-parton final state modified by process-independent terms:

$$
\mathrm{d} \sigma_{n+1}=\mathrm{d} \sigma_{n} \frac{\mathrm{d} t \mathrm{~d} z}{t} \frac{\alpha_{\mathrm{s}}\left(\mu^{2}\right)}{2 \pi} \hat{P}_{b a}(z) .
$$

Here $z$ is the energy or light-cone momentum fraction taken by the outgoing parton, and

the splitting kernel $\hat{P}_{b a}(z)$ typically is given by the (unregularised) Altarelli-Parisi splitting function or kernels that reduce to it in the collinear limit. The parameter $t \approx k_{\perp}^{2} \approx Q^{2}$ or, alternatively, $t \approx \theta^{2}$ is a measure for the hardness of the additional emission, to leading logarithmic accuracy this can be the square of the transverse momentum $k_{\perp}$, the virtual mass $Q$ or the emission angle $\theta$. The scale $\mu^{2}$ at which the strong coupling $\alpha_{\mathrm{s}}$ is evaluated is usually given by $\mu^{2}=k_{\perp}^{2}$. While eq. (2.1) captures all leading collinear and soft-collinear logarithms irrespective of the precise definition of $t$, the choice $t=\theta^{2}$ and the identification 
$\mu^{2}=k_{\perp}^{2}$ ensure that also the next-to leading logarithmic contributions are properly taken into account. ${ }^{2}$

Closer inspection of eq. (2.1) reveals that the cross section diverges for $t \rightarrow 0$, as expected. However, emissions at low scales $t$ of the order of a few $\Lambda_{\mathrm{QCD}}$ will typically not yield any resolvable parton, instead partons radiated at such scales will most likely end up in the same hadron as their emitter. This justifies the introduction of an infrared cut-off $t_{\mathrm{c}}$, usually chosen such that the transverse momentum is cut at $k_{\perp}^{2}\left(t_{\mathrm{c}}\right) \approx 1 \mathrm{GeV}^{2}$. This cut-off of course also implies a cut-off in $z$ thus shielding the divergent structures inside the splitting kernels $\hat{P}$.

Iterating eq. (2.1) for any number $k$ of additional emissions with these choices, such that

$$
\mathrm{d} \sigma_{n+k}=\mathrm{d} \sigma_{n} \prod_{j=1}^{k} \frac{\mathrm{d} t_{j} \mathrm{~d} z_{j}}{t_{j}} \frac{\alpha_{\mathrm{s}}\left(k_{\perp, j}^{2}\right)}{2 \pi} \hat{P}_{j}\left(z_{j}\right) \Theta\left(t_{j-1}-t_{j}\right),
$$

thus resums, to all orders, terms of the parametric form $\alpha_{\mathrm{s}}^{l}\left[L^{2 l}+L^{2 l-1}\right]$, where $L$ denotes large logarithms of ratios of the scales, $t_{0} / t_{\mathrm{c}}$. It should be noted that this accuracy is achieved in the limit, where the number of colours is infinite, $N_{c} \rightarrow \infty$. Configurations that are of sub-leading colour are typically suppressed with $1 / N_{c}^{2}$ and come at order $\alpha_{\mathrm{s}}^{l} L^{2 l-1}$ or below.

The picture developed so far treats emissions as independent from each other (interferences between subsequent emissions are sub-leading) and independent from the hard process, apart from the strict ordering $t_{\mathrm{h}}=t_{0}>t_{1}>t_{2}>\ldots$ indicated by the $\Theta$-function in eq. (2.2) and apart from the exact definition of the scale $t_{\mathrm{h}}=t_{0}$, which indeed depends on the process. However, it is worth noting that the choices of $t \approx \theta^{2}$ and $t \approx k_{\perp}^{2}$ emerge as better suited when inspecting the radiation pattern of two subsequent emissions since they better capture effects related to the emergence of destructive interference in non-angular-ordered emissions [4, 59, 60].

The picture above can be cast into a probabilistic form, optimally suited for detailed simulation by introducing the Sudakov form factor,

$$
\mathcal{S}_{a}\left(t_{\mathrm{h}}, t_{\mathrm{c}}\right)=\exp \left\{-\int_{t_{\mathrm{c}}}^{t_{\mathrm{h}}} \frac{\mathrm{d} t}{t} \int_{z_{\min }}^{z_{\max }} \mathrm{d} z \sum_{b} \frac{\alpha_{\mathrm{s}}\left(k_{\perp}^{2}\right)}{2 \pi} \hat{P}_{b a}(z)\right\} .
$$

It can be interpreted as the probability that a parton $a$ emits no resolvable radiation between the starting scale $t_{\mathrm{h}}$ and the cut-off $t_{\mathrm{c}}$. The independence of the emissions guarantees that the resulting simulation, the parton shower, can be represented as a Markov chain, i.e. the probability for any further emission depends only on the current state of the parton ensemble and not on the history leading to it. For the generation of radiation off final state particles the parton shower starts at the scale of the hard core process, each further emissions has to be softer than the previous until the infrared cut-off is reached.

The fact that the result of the evolution is fixed by the hard process at the higher and by the known initial state, the incident hadron, at the lower scale complicates the

\footnotetext{
${ }^{2}$ In dipole-like showers, also the choice $t=k_{\perp}^{2}$ appears to allow for a resummation of such next-to leading logarithms [58].
} 
generation of emissions off initial state particles. It would thus be very inefficient to evolve from the soft, hadronic scale up to the hard scale and discard all histories that do not match the hard process. Instead, initial state partons are evolved backwards starting from the hard process with the parton density at each evolution step constrained by the parton distribution functions (PDFs). This modifies the Sudakov form factor such that

$$
\mathcal{S}_{a}^{(\mathrm{IS})}\left(t_{\mathrm{h}}, t_{\mathrm{c}}, x\right)=\frac{f\left(x, t_{\mathrm{c}}\right)}{f\left(x, t_{\mathrm{h}}\right)} \mathcal{S}_{a}\left(t_{\mathrm{h}}, t_{\mathrm{c}}\right) .
$$

Written in such a form, with suitable Sudakov form factors, the parton shower correctly resums all leading and dominant sub-leading logarithms. Various methods to further improve the accuracy of the parton shower away from the soft and collinear limits of secondary parton radiation exist. However, since here we are mainly concerned with the properties of jets, it is sufficient to stress that the parton shower, due to its logarithmic accuracy, correctly describes the bulk of all QCD events, and in particular the properties of jets, which are characterised by multiple soft and collinear emissions.

\subsection{Scattering in a medium and parton showers}

To analyse how medium effects enter the picture, consider a single scattering of an energetic projectile off a parton in the medium at a time that is late enough such that the parton shower evolution of the initial hard scattering process is finished and all emerging final state partons are on their mass shell. This process fundamentally is not different from the hard initial process that produced the hard parton in the first place. Only the centre-of-mass energy and thus the scale of the scattering are, on average, diminished and since there is no natural infrared cut-off as in the case of the initial hard scatter (the criterion that the jets should have at least a certain $p_{\perp}$ ) one has to specify how the infrared region is to be treated. In JEWEL an infrared continuation of the matrix elements is introduced, which will be discussed in section 3. Then, just as in the case of the initial hard jet production process, leading order $2 \rightarrow 2$ matrix elements supplemented with parton showers provide a powerful approximation to higher-order real emission matrix elements.

The only difference with respect to the case already discussed in section 2.1 relevant for the implementation of such a picture is that the incident partons in such a secondary scatter are not constituents of an incoming nucleon. Therefore, using nucleon PDFs in the initial state parton shower is inappropriate and instead 'partonic PDFs' must be invoked to properly account for the fact that in the backwards evolution of the initial state shower the incident particles are known. These parton PDFs are constructed to describe the densities of partons in partons, by taking into account possible radiation above the resolution scale $t_{\mathrm{c}}$. At and below $t_{\mathrm{c}}$ no radiation is possible and the parton has no associated parton density. The partonic PDFs are thus computed by integrating the DGLAP equations with the boundary conditions

$$
f_{i}^{j}\left(x, t_{\mathrm{c}}\right)=\left\{\begin{array}{ll}
\delta(1-x) & ; i=j \\
0 & ; i \neq j
\end{array} .\right.
$$

In this way $2 \rightarrow 2$ configurations without additional radiation, that would be classified as elastic processes, and configurations with 3 and more final state particles, that would 
be regarded as inelastic processes or scattering with medium-induced Bremsstrahlung are generated at the same time, with no additional modelling bias. It should also be noted that matrix elements for different multiplicities of final state partons cannot be naively combined due to double counting issues [61], which are typically rarely addressed in the phenomenology of jet quenching effects. More importantly, though, these processes all come with the (leading log) correct relative rates (the total scattering rate is defined by the LO cross section as the parton shower is unitary). In principle, hard emissions could also be corrected to the full matrix elements using merging prescriptions such as [61, 62], but for the purpose of this study the accuracy of the parton shower is sufficient. A further advantage of this description is that it is not restricted to eikonal or close-to-eikonal kinematics (coherence issues will be discussed in section 3.5).

A complication arises when a rescattering occurs before the parton shower evolution of the previous scattering (initial or rescattering in the medium) has terminated. The interplay of such different sources of radiation in JEWEL is governed by formation times. The formation time associated with an emission parametrically is of the form

$$
\tau=\frac{E}{t},
$$

where $E$ is the emitting parton's energy. ${ }^{3}$ In the case of rescattering during the parton shower evolution, it is the emission with the shorter formation time that is formed, while the other emission will be discarded. This means in turn that sufficiently hard rescatterings reset the parton shower and restart it at a scale given by their kinematics, while soft rescatterings cannot initiate further radiation.

To further illustrate these points, consider a situation where a parton in the parton shower (it does not matter which scattering initiated this parton shower) is going to split at a scale $t_{1}$ on a timescale $\tau_{1}$, while a further scattering with scale $t_{2}^{(\mathrm{s})}$ just happens before that. Then a hypothetical parton shower with starting scale $t_{2}^{(\mathrm{s})}$ is initiated. If the hypothetical parton shower does not radiate, then the original splitting at $t_{1}$ proceeds as foreseen. If, on the other hand, the hypothetical parton shower would initiate radiation at a scale $t_{2}$ with corresponding formation time $\tau_{2}$, then there are two possible outcomes: If $\tau_{2}>\tau_{1}$ the hypothetical shower is discarded and only the splitting at $t_{1}$ happens. In the opposite case $\tau_{2}<\tau_{1}$ the original splitting at $t_{1}$ is discarded, the hypothetical shower becomes real and replaces the original one. The splitting at $\tau_{2}$ takes place and any further radiation from the new parton shower proceeds, provided it is not disturbed by further rescattering. This formation time prescription is on average equivalent to the statement that only rescatterings which are harder than the current parton shower scale can resolve the virtual parton. As a consequence, individual emissions cannot be unambiguously associated with a certain scattering.

This is the algorithm for incoherent emission. The LPM interference was not considered yet, its implementation in a probabilistic framework will be discussed in sections 2.3 and 3.5. Taking the LPM effect into account does, however, not change the structure of the

\footnotetext{
${ }^{3}$ This is parametrically equivalent to the form $\tau=2 \omega / k_{\perp}^{2}$, familiar from analytical calculations of coherent Bremsstrahlung in the eikonal limit.
} 
algorithm. It merely allows to replace a single momentum transfer in the above discussion with the coherent sum of several scatterings with an effective momentum transfer. The details are given in section 3.5.

\subsection{Reminder: probabilistic interpretation of the LPM effect}

Analytical calculations of medium induced gluon radiation in the eikonal limit found that the non-Abelian analogue of the Landau-Pomeranchuk-Migdal (LPM) effect plays an important role. This is a destructive interference between emissions initiated by subsequent scattering processes that occurs when the formation times of the individual emissions overlap. In [47] a probabilistic formulation suitable for Monte Carlo implementation was derived, the findings relevant for this discussion will be summarised here.

In the eikonal limit, the radiating parton has asymptotically high energy $E$. The medium constituents then appear as static scattering centres and the momentum transfer between the projectile and the scattering centres is purely transverse. The original BDMPS approach [34] and similar other approaches [35-39] operate in a kinematical regime where $E \gg \omega \gg k_{\perp}, q_{\perp}$, where $\omega$ is the gluon energy, $k_{\perp}$ denotes the transverse momentum of the gluon and $q_{\perp}$ the momentum transfer from the scattering centre. ${ }^{4}$ Gluon radiation is described by a Gunion-Bertsch cross section of the form

$$
\frac{\mathrm{d} \sigma^{(\mathrm{GB})}}{\mathrm{d} \mathbf{k}_{\perp} \mathrm{d} \mathbf{q}_{\perp}} \propto \frac{\mathbf{q}_{\perp}^{2}}{\mathbf{k}_{\perp}^{2}\left(\mathbf{k}_{\perp}-\mathbf{q}_{\perp}\right)^{2}} .
$$

For the sake of deriving and validating a probabilistic interpretation of gluon radiation and in particular the LPM-effect it is entirely sufficient to keep only the singular part (i.e. set $\mathbf{k}_{\perp}=\mathbf{q}_{\perp}$ ) and ascribe all elastic rescatterings to the gluon (as only the transverse momentum of the gluon relative to the projectile is relevant).

A radiated gluon decoheres from the projectile when it has accumulated a relative phase

$$
\varphi=\frac{k_{\perp}^{2}}{2 \omega} \Delta L
$$

of order unity. Here, $\Delta L$ is the distance it travelled. From this condition the gluon formation time $\tau=2 \omega / k_{\perp}^{2}$ can be read off.

Inspection of the calculations reveals that the scatterings occurring during the gluon formation time act coherently to radiate the gluon. In the gluon radiation term only the vector sum $\mathbf{Q}_{\perp}=\sum_{i} \mathbf{q}_{\perp, i}$ of the individual momentum transfers appears.

The probabilistic picture is that whenever an additional scattering occurs during the formation time, the respective momentum transfer is coherently added. There are two ways how this can be achieved in practice: The first one is to update the gluon phase accumulated up to the last scattering centre based on the gluon momentum before the last momentum transfer. Then the total $\mathbf{Q}_{\perp}$ including the last scattering is computed and the gluon $k_{\perp}$ is changed accordingly. The new formation time is determined taking the

\footnotetext{
${ }^{4}$ The BDMPS-Z formula for parton energy loss also holds in the kinematical region $\omega \gg E-\omega \gg k_{\perp}, q_{\perp}$, but an explicit derivation without strong energy ordering is not known. We thank P. Arnold for this comment.
} 
already existing phase into account. The other option takes the multiple scattering during the formation time less literally. In this case, whenever a further momentum transfer is added, the new $\mathbf{Q}_{\perp}$ and $k_{\perp}$ are computed and the gluon formation time given by the updated $k_{\perp}$ is calculated. If the last scattering still is within the updated formation time limit (counted from the first scattering) it is accepted, otherwise it is rejected as it leads to an inconsistent configuration. Finally, in both cases, when there are no more coherent momentum transfers the gluon emission is accepted with probability $1 / N_{\text {scat }}$, where $N_{\text {scat }}$ is the number of coherent scatterings.

\section{Details of the implementation}

\subsection{Event generation}

In our model, event generation proceeds as follows:

1. The hard matrix elements initially producing the di-jets and the corresponding initial state parton shower are generated by PYтнIA 6.4 [63] running with the virtualityordered parton shower to provide the best fit to the JEWEL simulation. There are no multi-parton interactions and the CTEQ6L1 [64] PDFs as provided by LHAPDF [65] are used; simulating nuclear collisions the nuclear modification of EPS09LO [66] is employed.

2. Then JEWEL takes over and selects the impact parameter of the event according to the geometrical cross section and the transverse position of the hard scattering according to the density of binary nucleon-nucleon interactions in the transverse plane. It then generates the final state parton showers including interactions in the medium. This includes rescatterings, emission of secondary partons, an implementation of the LPM effects and so on. These details will all be discussed below.

3. Finally, the event is handed back to PYTHIA for hadronisation.

Note that in the absence of a medium the procedure is the same and the JEwEL parton shower becomes an ordinary vacuum parton shower. This option is used for the validation of the JEWEL parton shower, for the generation of the $\mathrm{p}+\mathrm{p}$ baseline, and for the fixing of shower and hadronisation parameters.

In the following we will highlight aspects of phase 2 of the sketch above.

\subsection{In-medium matrix elements and their infrared continuation}

The interaction of the parton shower with the partonic constituents of the medium depends on properties of the medium. We specify the cross sections for $2 \rightarrow 2$ processes as

$$
\sigma_{i}(E, T)=\int_{0}^{|\hat{t}|_{\max }(E, T)} \mathrm{d}|\hat{t}| \int_{x_{\min }(|\hat{t}|)}^{x_{\max }(|\hat{t}|)} \mathrm{d} x \sum_{j \in\{\mathrm{q}, \overline{\mathrm{q}}, \mathrm{g}\}} f_{j}^{i}(x, \hat{t}) \frac{\mathrm{d} \hat{\sigma}_{j}}{\mathrm{~d} \hat{t}}(x \hat{s},|\hat{t}|),
$$

where the PDF takes into account possible initial state radiation off the energetic projectile. Note that here, implicitly, we neglect a similar evolution experienced by the target, i. e. 
initial state radiation off the medium parton. This choice, although inconsistent at first glance, is motivated by the fact that these partons have only very small energy in the rest frame of the medium, which translates into them being virtually unable to emit any resolvable parton.

The maximum momentum transfer $|\hat{t}|_{\max }$ is determined by the initial kinematics of the scattering. Neglecting the scattering centre's momentum $|\hat{t}|_{\max }=2 m_{\mathrm{s}}(T)\left[E_{\mathrm{p}}-m_{\mathrm{p}}\right]$, where $m_{\mathrm{s}}(T)$ stands for the (temperature dependent) scattering centre's mass and $E_{\mathrm{p}}$ and $m_{\mathrm{p}}$ are the projectile parton's energy and (virtual) mass, respectively. The boundaries on the $x$-integral are obtained from the requirement that $k_{\perp}^{2} \geq Q_{0}^{2} / 4$ and are given by $x_{\min }(|\hat{t}|)=Q_{0} /(4|\hat{t}|)$ and $x_{\max }(|\hat{t}|)=1-Q_{0} /(4|\hat{t}|)$. For the partonic cross section we keep leading terms only, but we regularise them with a Debye mass $\mu_{\mathrm{D}} \approx 3 T$. They therefore read

$$
\frac{\mathrm{d} \hat{\sigma}}{\mathrm{d} \hat{t}}(\hat{s},|\hat{t}|)=C_{\mathrm{R}} \frac{\pi}{\hat{s}^{2}} \alpha_{\mathrm{s}}^{2}\left(|\hat{t}|+\mu_{\mathrm{D}}^{2}\right) \frac{\hat{s}^{2}+(\hat{s}-|\hat{t}|)^{2}}{\left(|\hat{t}|+\mu_{\mathrm{D}}^{2}\right)^{2}} \longrightarrow C_{\mathrm{R}} 2 \pi \alpha_{\mathrm{s}}^{2}\left(|\hat{t}|+\mu_{\mathrm{D}}^{2}\right) \frac{1}{\left(|\hat{t}|+\mu_{\mathrm{D}}^{2}\right)^{2}} .
$$

\subsection{Parton shower}

The JEwEL parton shower [51] is a virtuality-ordered monopole shower, similar to the Pyтhia 6 virtuality ordered shower [63], but without applying, "by hand" the a posteriori angular ordering constraint, when being run in the medium. Identifying the evolution parameter $t$ with the virtual mass squared $Q^{2}$ of the partons in the shower, the infrared cut-off is denoted by $Q_{0}^{2}$, and the splitting parameter $z$ denotes the energy splitting. In this setup, the transverse momentum of the splitting is given by $k_{\perp}^{2} \simeq z(1-z) Q^{2}$ for time-like and $k_{\perp}^{2} \simeq(1-z) Q^{2}$ for space-like splittings. The current implementation treats all quarks as massless, the extension to massive quarks is straightforward.

Contrary to the way in which the PүтніA 6 shower is implemented, the kinematics of each splitting is already fully constructed when the splitting is generated. This requires determining the daughter virtualities together with the splitting of the mother. For parton shower evolution in the vacuum this procedure is more involved than the traditional approach of later correcting the kinematics of the splitting of the mother, but is advantageous for jet evolution in a medium. The reason for this is that in the medium one evolves not only in the shower ordering variable but also in time and going back to the mother splitting means going back in time, which is very cumbersome. The parton shower is completely democratic: all produced partons are allowed to split and - in case the parton shower develops in a medium - scatter themselves. This applies to all radiated partons, since there is anyway no way to unambiguously distinguish vacuum and medium-induced emissions. All partons are propagated through the medium following their own trajectories.

Since the centre-of-mass energies of scatterings in the medium are typically rather small, it is unlikely that more than one initial state splitting is initiated in secondary scatters. In this approximation ${ }^{5}$ the partonic PDFs can be integrated analytically yielding

$$
f_{\mathrm{q}}^{\mathrm{q}}\left(x, Q^{2}\right)=\mathcal{S}_{\mathrm{q}}\left(Q^{2}, Q_{0}^{2}\right) \delta(1-x)+\int_{Q_{0}^{2}}^{Q^{2}} \frac{\mathrm{d} q^{2}}{q^{2}} \mathcal{S}_{\mathrm{q}}\left(Q^{2}, q^{2}\right) \frac{\alpha_{\mathrm{s}}\left((1-x) q^{2}\right)}{2 \pi} \hat{P}_{\mathrm{qq}}(x),
$$

\footnotetext{
${ }^{5}$ The approximation used here can easily be improved numerically, adding more emissions, if necessary.
} 


$$
\begin{aligned}
& f_{\overline{\mathrm{q}}}^{\mathrm{q}}\left(x, Q^{2}\right)=0 \\
& f_{\mathrm{g}}^{\mathrm{q}}\left(x, Q^{2}\right)=\int_{Q_{0}^{2}}^{Q^{2}} \frac{\mathrm{d} q^{2}}{q^{2}} \mathcal{S}_{\mathrm{g}}\left(Q^{2}, q^{2}\right) \frac{\alpha_{\mathrm{s}}\left((1-x) q^{2}\right)}{2 \pi} \hat{P}_{\mathrm{gq}}(x), \\
& f_{\mathrm{q}}^{\mathrm{g}}\left(x, Q^{2}\right)=f_{\overline{\mathrm{q}}}^{\mathrm{g}}\left(x, Q^{2}\right)=\int_{Q_{0}^{2}}^{Q^{2}} \frac{\mathrm{d} q^{2}}{q^{2}} \mathcal{S}_{\mathrm{q}}\left(Q^{2}, q^{2}\right) \frac{\alpha_{\mathrm{s}}\left((1-x) q^{2}\right)}{2 \pi} \hat{P}_{\mathrm{qg}}(x), \\
& f_{\mathrm{g}}^{\mathrm{g}}\left(x, Q^{2}\right)=\mathcal{S}_{\mathrm{g}}\left(Q^{2}, Q_{0}^{2}\right) \delta(1-x)+2 \int_{Q_{0}^{2}}^{Q^{2}} \frac{\mathrm{d} q^{2}}{q^{2}} \mathcal{S}_{\mathrm{g}}\left(Q^{2}, q^{2}\right) \frac{\alpha_{\mathrm{s}}\left((1-x) q^{2}\right)}{2 \pi} \hat{P}_{\mathrm{gg}}(x) .
\end{aligned}
$$

\subsection{Time evolution}

Positions and times in JEWEL are measured in the (global) rest frame of the medium. For each parton in the simulation, the question whether, when and where the next scattering will occur can be answered by evaluating a local scattering rate, see e.g. [51]. In JEwEL, the corresponding local scattering rate is the product of the cross section $\sigma_{i}(E, T)$ of eq. (3.1) and the local density of scattering centres. We note that this definition of the local scattering rate allows naturally for the possibility that parton branchings are initiated before scattering. Once JEWEL decides that a scattering will take place, the kinematics of this scattering is determined according to the differential cross section eq. (3.2).

The medium model used in the present study for the local density of scattering centers is described in more detail in the subsection 3.7. We emphasize that the time evolution implemented in JEWEL can be interfaced with all medium models that characterise the medium as a phase space distribution of partonic scattering centres.

The time evolution described here specifies for each parton in the simulation whether it interacts with the medium, and if so, at which spatio-temporal position and with which kinematics. To what extent spatio-temporally neighboring interactions with the medium act coherently is decided by the conditions to which we turn now.

\subsection{Implementation of the LPM effect in JEWEL}

In JEWEL it is assumed that the physical reasoning behind the probabilistic formulation of the LPM-effect is valid also outside the eikonal limit. Although the kinematics is very different, the procedure of adding scattering processes within the gluon formation time coherently by adding their momentum transfers and reweighting the actual emissions proceeds in exactly the same way.

A slight complication arises due to the fact, that - in contrast to the eikonal case the formation time is not directly related to the momentum transfer but to the scale of the emission, which can be anything between the scale $t^{(\mathrm{s})}$ set by the momentum transfer and $t_{\mathrm{c}}$. In JEWEL, formation time and emission scale are two aspects of a single algorithmic procedure. In the following, we discuss the implementation of the LPM effect by addressing first how the scale of the emission can change dynamically, and by clarifying then the operational role of the formation time introduced in section 2.2. The procedure for adding 
a momentum transfer coherently and determining the scale of the associated radiation is outlined below. Here, $t_{1}^{(\mathrm{s})}$ denotes the scale of scattering before that last momentum transfer is added and $t_{2}^{(\mathrm{s})}$ is the scale including the last momentum transfer. As the momentum transfers are added vectorially, the resulting $t_{2}^{(\mathrm{s})}$ may be larger or smaller than $t_{1}^{(\mathrm{s})}$. Before the last scattering, $t_{1}^{(\mathrm{s})}$ was the starting scale of a parton shower and (provided $t_{1}^{(\mathrm{s})}>t_{\mathrm{c}}$ ) the parton shower may or may not foresee a gluon radiation at a scale $t_{1}<t_{1}^{(\mathrm{s})}$. Updating the starting scale set by the effective (coherent) scatterings to $t_{2}^{(\mathrm{s})}$ may increase or decrease the phase space for radiation and the existing emission (if there is one) has to be corrected for the corresponding change in phase space and emission probability. As a result an emission may have to be rejected, it may be assigned a new scale $t_{2}$, or a new emission may be enforced where there was none before. All the different cases are summarised below.

- $t_{2}^{(\mathrm{s})}<t_{\mathrm{c}}$ :

In this situation no radiation is possible, if there is an existing emission it has to be rejected.

- existing radiation, $t_{2}^{(\mathrm{s})}>t_{1}^{(\mathrm{s})}$ :

The probability for an emission is now larger and so the existing radiation cannot be rejected. The scale of the radiation, however, may have to be redetermined. The chosen $t_{1}$ for the emission is kept with probability $\mathcal{S}\left(t_{2}^{(\mathrm{s})}, t_{1}^{(\mathrm{s})}\right)$, i.e. if there is no radiation between $t_{1}^{(\mathrm{s})}$ and $t_{2}^{(\mathrm{s})}$ it has to be in the old interval $\left[t_{\mathrm{c}}, t_{1}^{(\mathrm{s})}\right]$. In the other case where we have radiation between $t_{1}^{(\mathrm{s})}$ and $t_{2}^{(\mathrm{s})}$ a new scale $t_{2}$ in this interval is chosen and the old one has to be discarded.

- existing radiation, $t_{1}^{(\mathrm{s})}>t_{2}^{(\mathrm{s})}>t_{\mathrm{c}}$ :

In this case the probability for radiation has decreased and the existing emission has to be rejected with probability $\left[1-\mathcal{S}\left(t_{1}^{(\mathrm{s})}, t_{\mathrm{c}}\right)\right] /\left[1-\mathcal{S}\left(t_{2}^{(\mathrm{s})}, t_{\mathrm{c}}\right)\right]$. If the emission is kept but its scale $t_{1}$ is larger than $t_{2}^{(\mathrm{s})}$ a new scale $t_{2}$ in the new interval has to be chosen.

- no existing radiation, $t_{2}^{(\mathrm{s})}>t_{1}^{(\mathrm{s})}$ :

The phase space for emission has increased and with probability $1-\mathcal{S}\left(t_{2}^{(\mathrm{s})}, t_{1}^{(\mathrm{s})}\right)$ a new emission with scale $t_{2}$ to be chosen between $t_{1}^{(\mathrm{s})}$ and $t_{2}^{(\mathrm{s})}$ is added.

- no existing radiation, $t_{1}^{(\mathrm{s})}>t_{2}^{(\mathrm{s})}>t_{\mathrm{c}}$ :

In this case nothing happens since the probability for radiation has decreased and no emission needs to be reweighted.

Consistent with our discussion in section 2.3, for all cases with an emission at scale $t$ this emission is assigned a formation time $\tau=E / t$ where $E$ is the emitting parton's energy. ${ }^{6}$ Within this formation time, the algorithm searches then for further momentum transfers from the medium, that - if found - are added coherently and will change the radiation phase space, which can lead to a change in the scale $t$ (and thus the formation time) at which the emission actually happens. At the end of this process, when no further

\footnotetext{
${ }^{6}$ In fact, the formation times are obtained from an exponential distribution, whose mean value is $\tau=E / t$.
} 
momentum transfer within the dynamically adjusted formation time is found, the emission is reweighted with a factor $1 / N_{\text {scat }}$ as discussed in section 2.3. In JEWEL, emissions are only accepted if their final formation time is shorter than that of potential other emissions (cf. section 2.2).

\subsection{Recoils, colour flows, and hadronisation}

In JEWEL only the QCD evolution of jets and their interactions with the medium are simulated and not the complete event. The recoiling scattering centres can be traced and in principle they could be subjected to further interactions. This option is, however, disabled by default as these interactions are typically much softer than the jet-medium interactions and perturbation theory is not necessarily the correct language to describe them (and due to the rapid increase in number of interactions the run-time increases dramatically). As a way of estimating the effect of the jet on the medium and jet-background correlations the recoils may be kept in the event, removed or replaced with uncorrelated scattering centres.

As in the vacuum parton shower every radiated gluon is a colour neighbour of the emitter. When the recoils are not traced they also don't exchange colour with the jet. When the recoils are kept in the event, they are assumed to be neighbours of their scattering partner and are inserted in the existing colour string. This obviously constitutes a simplification but avoids another complication. After all splittings and scatterings the event is hadronised using PYTHIA's implementation of the Lund string fragmentation. For the Lund string model to work properly it is essential that the strings are of sufficient invariant mass. Naively trying to track all colour indices of all scattering centres without invoking any colour reconnection model indeed leads to large numbers of strings with very low invariant mass and the breakdown of the hadronisation model.

\subsection{Medium model}

JEWEL can in principle be interfaced with any medium model that specifies the density and momentum distribution of scattering centres for any point in space-time. In order to have a simple, highly predictive model capturing all essential features of the medium without introducing too much additional, potentially intractable kinematics and, at the same time, allowing full control over the medium, we used a variant of the Bjorken model $[67,68]$ for this study. It describes the boost-invariant longitudinal expansion of an ideal quark-gluongas with three parameters only, namely the initial proper time $\tau_{\mathrm{i}}$ at which the hydrodynamic evolution sets in, the initial temperature $T_{\mathrm{i}}=T\left(\tau_{\mathrm{i}}\right)$ and the critical temperature $T_{\mathrm{c}}$ of the deconfinement phase transition. The transverse profile is chosen such that the energy density is proportional to the density of wounded nucleons. The latter is, as all geometrical aspects, calculated in the framework of a simple Glauber model [69]. In practice this means that the initial temperature is translated into an initial energy density $\epsilon_{\mathrm{i}} \propto T_{\mathrm{i}}^{4}$. The actual energy density profile at $\tau_{\mathrm{i}}$ is then given by

$$
\epsilon\left(x, y, b, \tau_{\mathrm{i}}\right)=\epsilon_{\mathrm{i}} \frac{n_{\text {part }}(x, y, b)}{\left\langle n_{\text {part }}\right\rangle(b=0)} \quad \text { with } \quad\left\langle n_{\text {part }}\right\rangle(b=0) \approx \frac{2 A}{\pi R_{A}},
$$

where $b$ is the impact parameter, $n_{\text {part }}(x, y, b)$ is the density of participating nucleons in the transverse plane and we have for simplicity assumed a symmetric $A+A$ collision. As a 

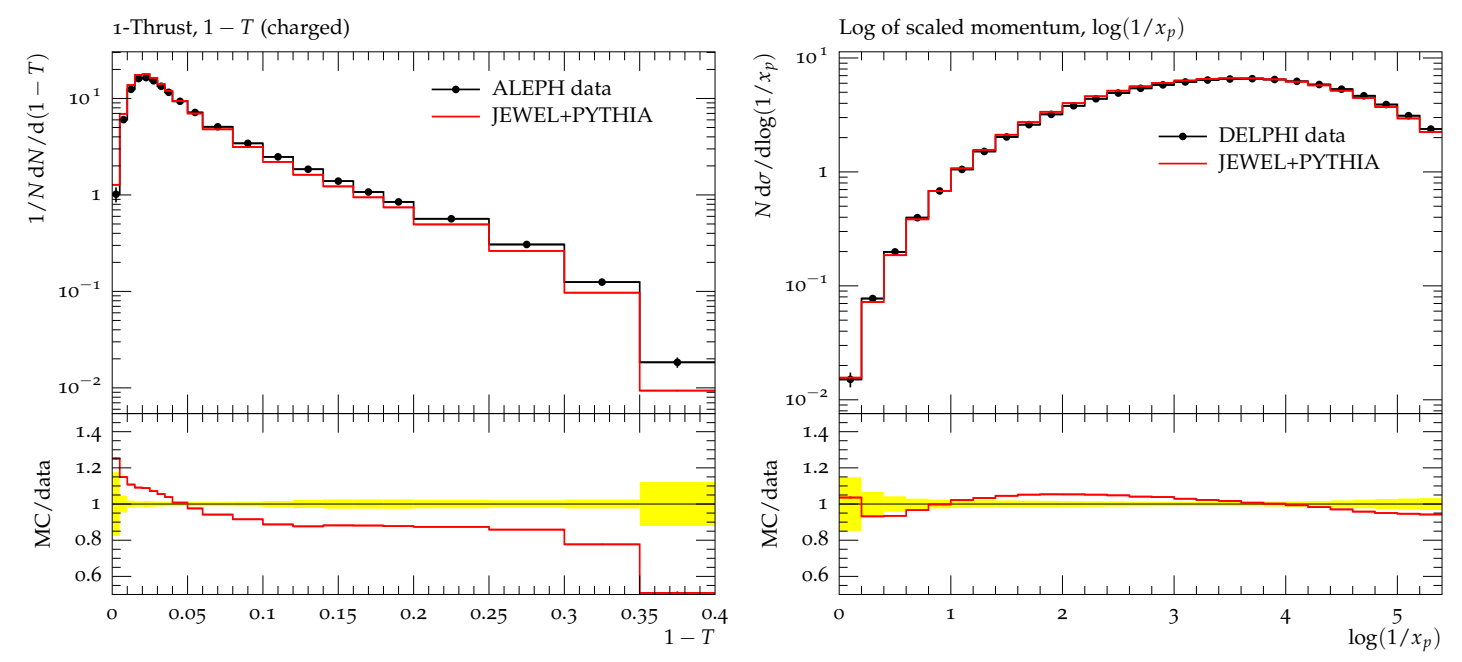

Figure 1. Comparison of Jewel+Pythia results to LeP data. L.h.s.: thrust distribution measured by ALEPH [71]. R.h.s.: charged particle fragmentation function as a function of the scaled momentum $x_{\mathrm{p}}=2 p_{\mathrm{h}} / \sqrt{s}$ measured by DELPHI [72].

consequence the temperature is higher in the centre of the overlap region and it increases with centrality. The (proper) time dependence is given by

$$
\epsilon(x, y, b, \tau)=\epsilon\left(x, y, b, \tau_{\mathrm{i}}\right)\left(\frac{\tau}{\tau_{\mathrm{i}}}\right)^{-4 / 3} \quad \text { and } \quad T(x, y, b, \tau) \propto \epsilon^{1 / 4}\left(x, y, b, \tau_{\mathrm{i}}\right)\left(\frac{\tau}{\tau_{\mathrm{i}}}\right)^{-1 / 3}
$$

The time dependence of the particle density is then given by $n(x, y, b, \tau) \propto T^{3}(x, y, b, \tau)$. We fix the critical temperature at $T_{\mathrm{c}}=165 \mathrm{MeV}$ consistent with lattice results and since we are only considering interactions in the deconfined phase there are no scatterings when the local temperature has dropped below $T_{\mathrm{c}}$.

\section{Results}

The analysis of Monte Carlo events and all plots shown here were produced with Rivet [70] and the corresponding analysis codes contained within. For jets, typically the anti- $k_{\perp}$ algorithm of [23] has been used throughout.

\subsection{Validation}

In the absence of a medium JEwEL reduces to a standard (vacuum) parton shower, which was validated extensively against data from LEP and $p+p$ collisions at LHC.

In all results shown here, the strong coupling is running at one loop with $\alpha_{\mathrm{s}}\left(m_{\mathrm{Z}}\right)=$ 0.128 , consistent with findings of other leading order parton shower calculations. The infrared cut-off of the parton shower was chosen as $Q_{0}=1.5 \mathrm{GeV}$. As the JeweL parton shower is very similar to the PYTHIA virtuality-ordered shower and given the very reasonable description of the LEP data, a retuning of the hadronisation parameters was considered not to be necessary. 

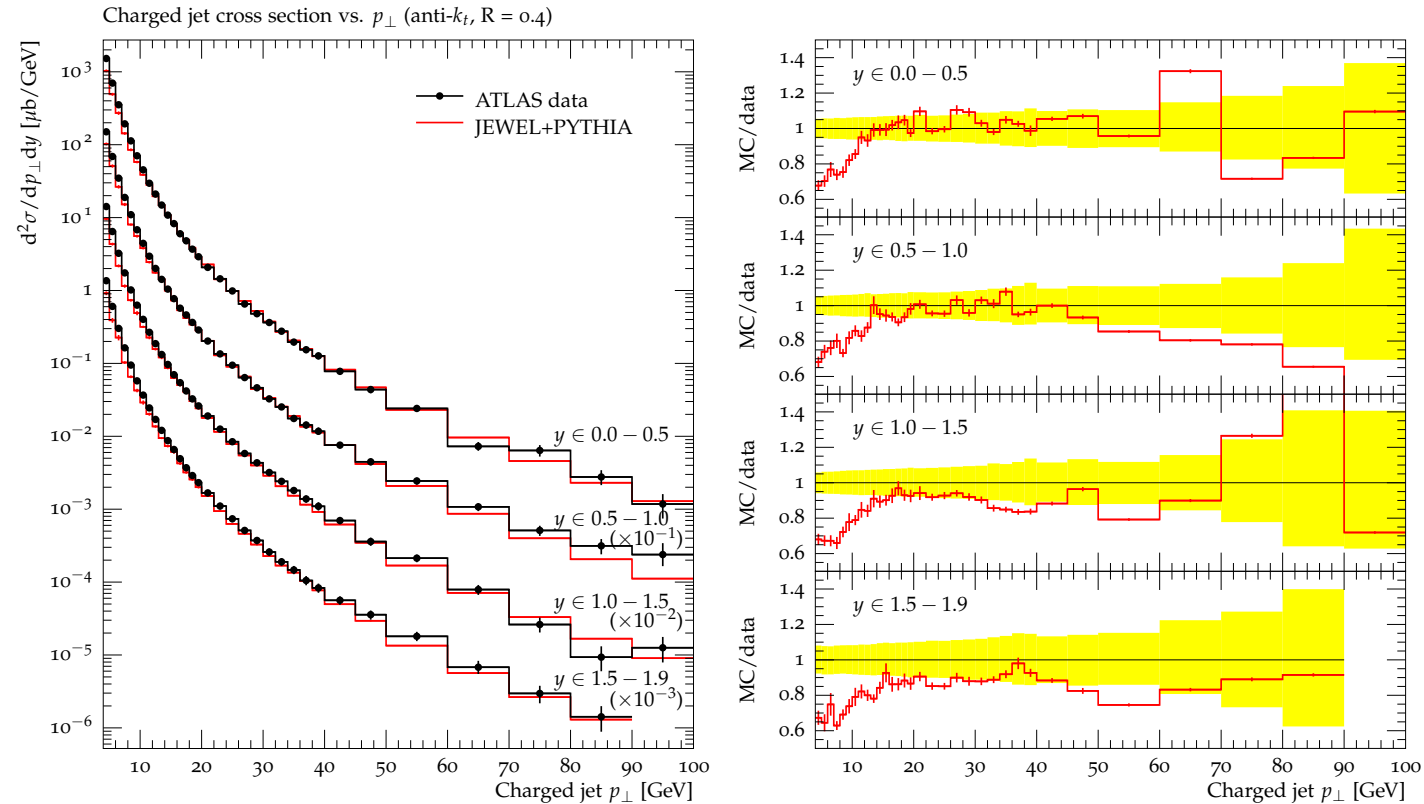

Figure 2. JeWEL + Pythia results for the inclusive jet cross section in different rapidity bins compared to ATLAS data [73] in $\mathrm{p}+\mathrm{p}$ at $\sqrt{s}=7 \mathrm{TeV}$. Jets are reconstructed using the anti- $k_{\perp}$ algorithm with $\mathrm{R}=0.4$ on tracks.
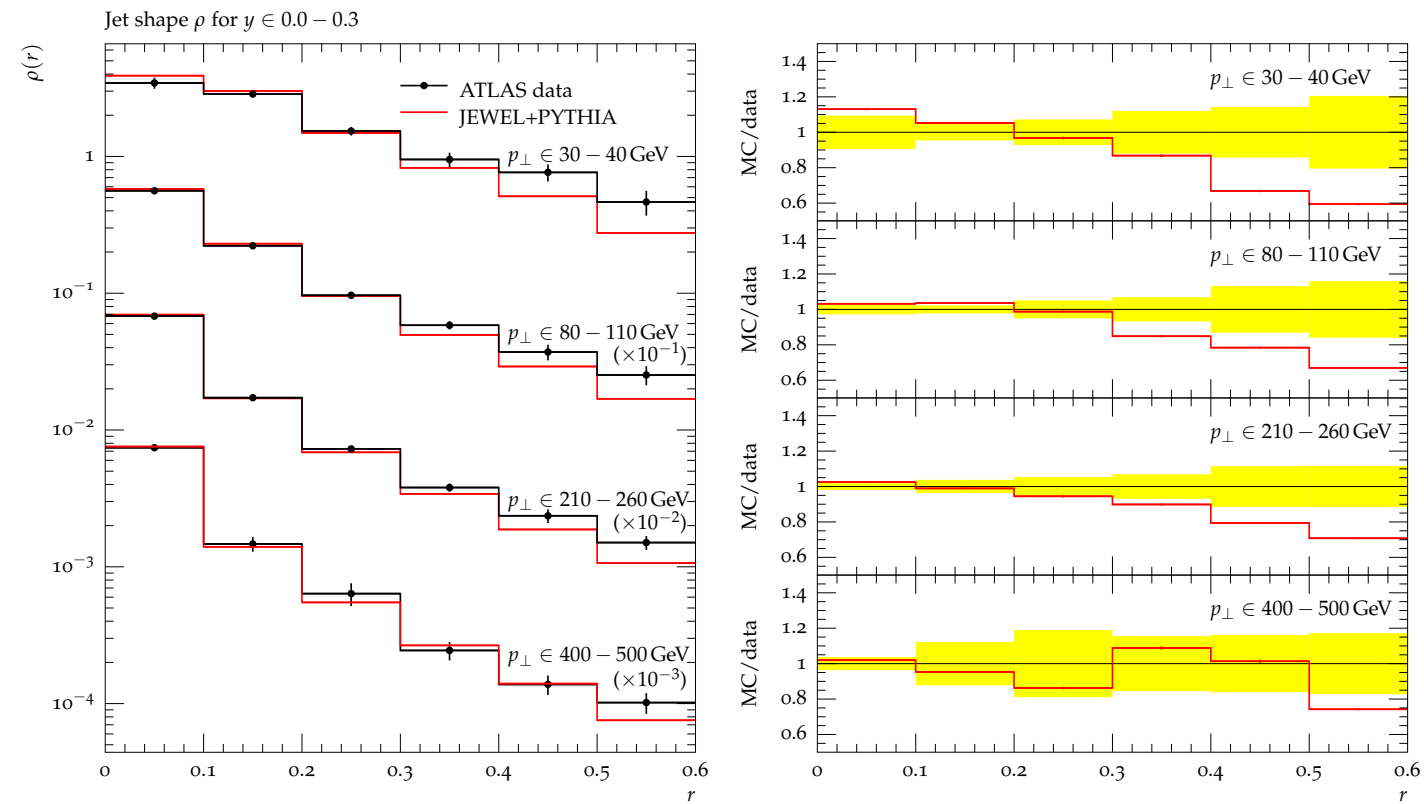

Figure 3. JeWEL + Pythia compared to AtLAs measurements of the jet shape $\rho(r)[74]$ in $\mathrm{p}+\mathrm{p}$ at $\sqrt{s}=7 \mathrm{TeV}$.

As examples illustrating the generally very satisfactory performance of the parton shower (and hadronisation) at LEP the thrust distribution and the charged particle fragmentation function are shown in figure 1 .

At the LHC the comparison of JEWEL+PYTHIA results to the jet data is complicated by the underlying event, which is not included in the MC. This can, for instance, be 

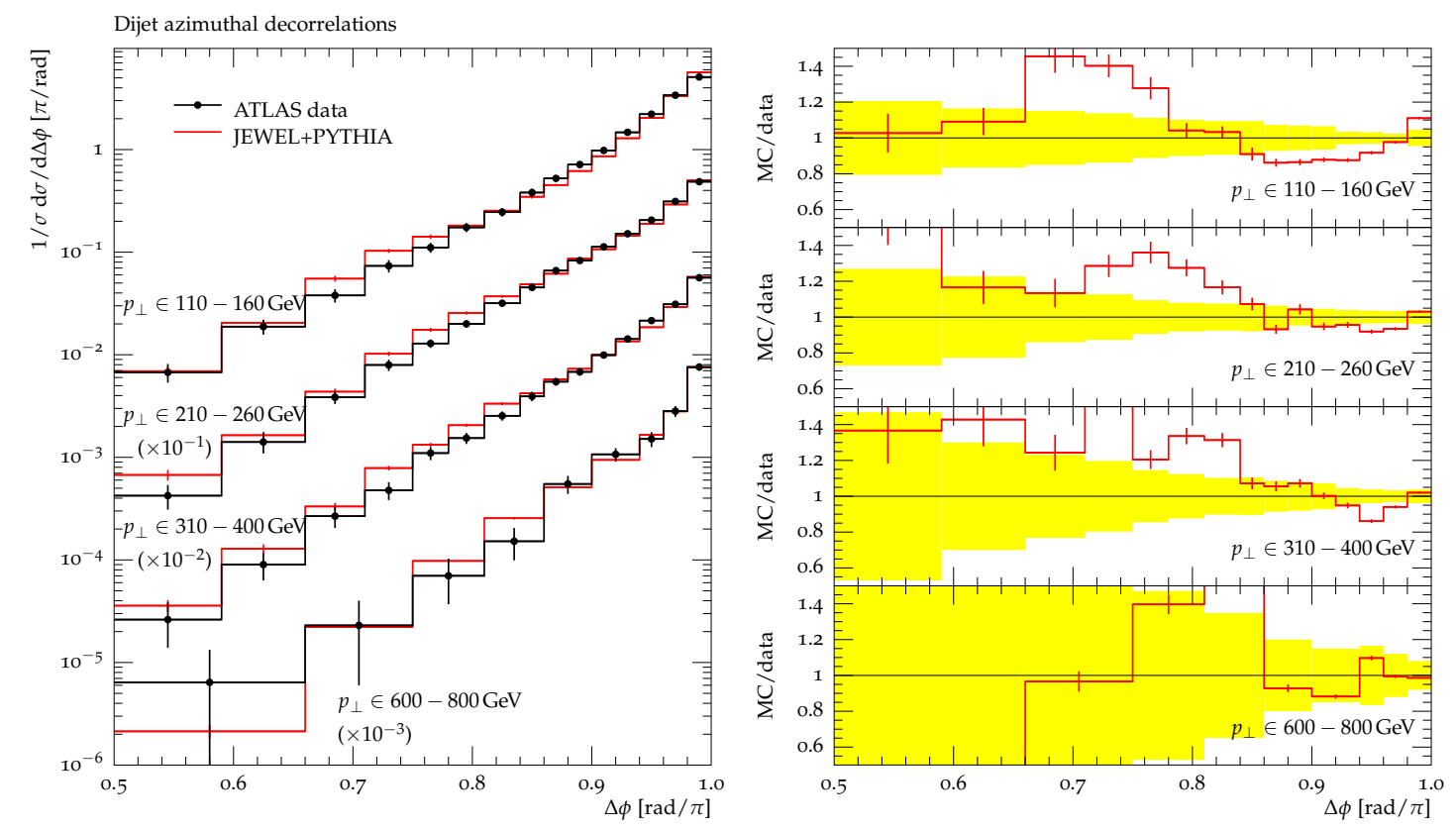

Figure 4. Jewel + Pythia compared to AtLas jet measurements of the azimuthal decorrelation [75] in $\mathrm{p}+\mathrm{p}$ at $\sqrt{s}=7 \mathrm{TeV}$.
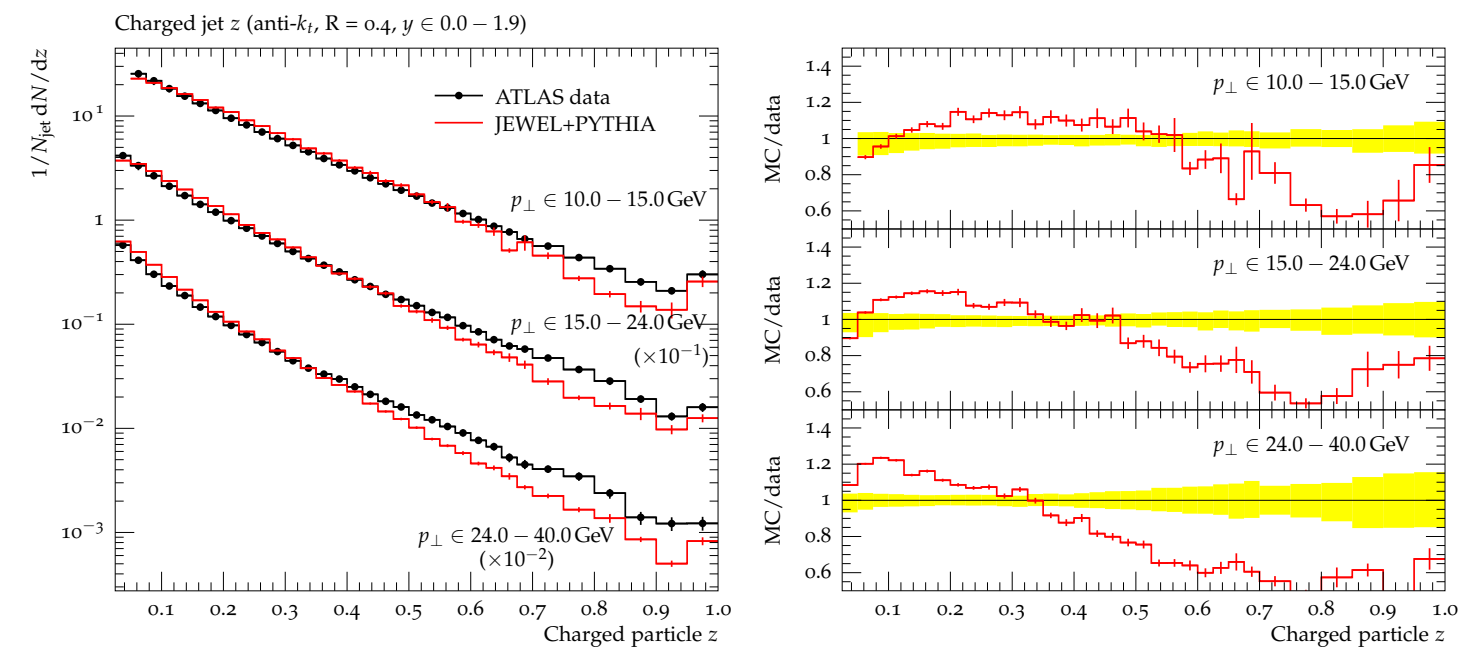

Figure 5. JeWEL + PythiA results for the fragmentation function of charged particles in track jets for different jet- $p_{\perp}$ bins compared to ATLAS data [73].

seen in the inclusive (track) jet cross section shown in figure 2: While the agreement between the MC results and the data is satisfactory at high $p_{\perp}$, the $\mathrm{MC}$ falls below the data at relatively small transverse momenta, where the contribution from the underlying event is largest. The same effect is visible in the (calorimetric) jet shape measurements (figure 3), where the JEWEL+PYTHIA jet are significantly more collimated at low $p_{\perp}$ and again the agreement improves with increasing $p_{\perp}$. A complementary observable, namely the azimuthal decorrelation shown in figure 4 , is also described reasonably well. 

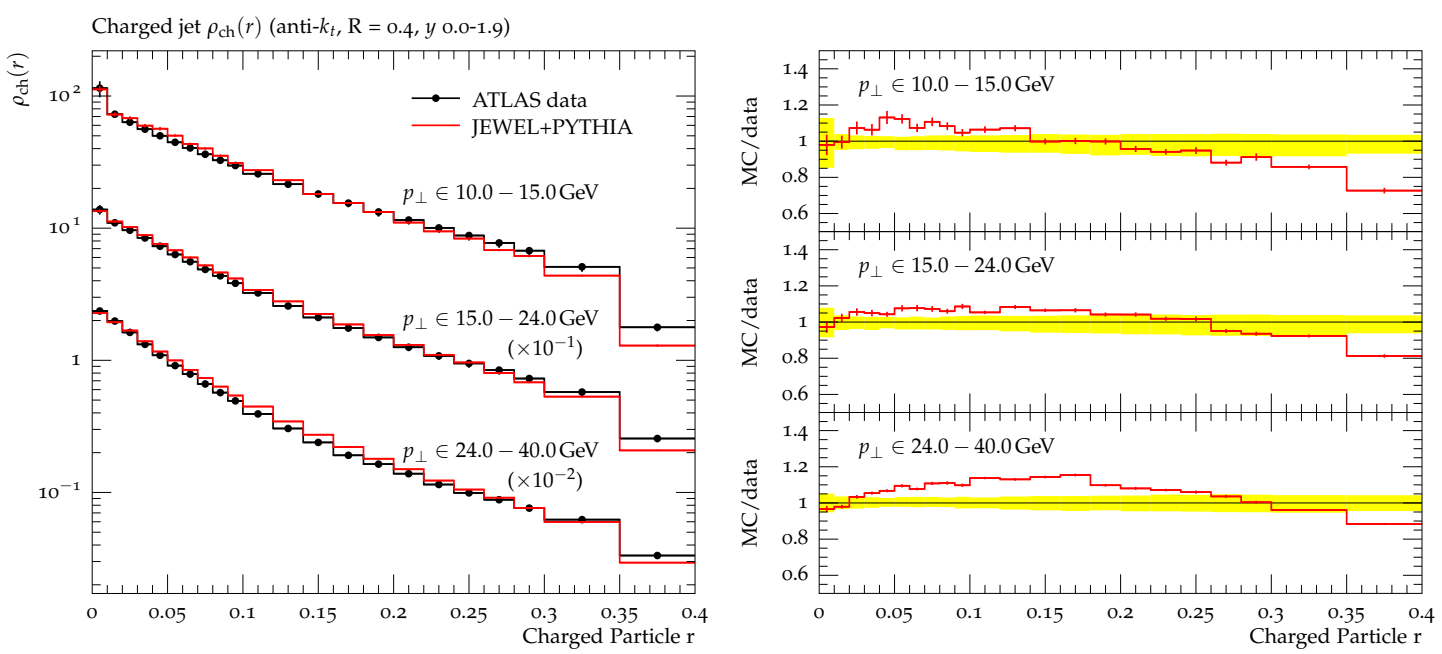

Figure 6. JeWEL + Pythia results for the charged particle density $\rho_{\text {ch }}(r)$ in track jets for different jet- $p_{\perp}$ bins compared to ATLAS data [73].

Figure 5 shows the comparison of Jewel+Pythia to the AtLas measurement of the charged particle fragmentation function in track jets. Here, the JEWEL+PyTHIA fragmentation consistently is too soft. The interpretation of this observation is, however, not straightforward, as the measurement is carried out for relatively low jet- $p_{\perp}$ and it is thus unclear to what extent there is a contamination from the underlying event. Two points hint at an issue in the MC in addition to possible underlying event contributions: Firstly, the charged particle density inside track jets shown in figure 6 shows better agreement between MC and data, although also here the components missing at large $r$ could be due to underlying event. Secondly, the jet shapes of calorimeter jets tend to be slightly too collimated in the MC even at high $p_{\perp}$ (figure 3), which would be consistent with a softer fragmentation function. It should be kept in mind that the jet shape $\rho(r)$ is an energy density, while $\rho_{\mathrm{ch}}(r)$ is a particle density.

The comparison of the JEwEL shower to the measurement of the ratio of the 3-jet to the 2-jet cross section by CMS shown in figure 7 demonstrates that the parton shower provides a very reasonable description of the 3 -jet matrix elements even well outside the collinear region. This confirms that leading order matrix elements in combination with a parton shower are a good approximation to radiative processes and thus provide a good estimate of the radiative energy loss.

Figure 8 shows a comparison with the neutral pion spectrum measured by PHENIX and the charged particle spectrum measured by CMS, which form the baseline for the respective measurements of the nuclear modification factor $R_{\mathrm{AA}}$. In the former case, above $p_{\perp} \simeq 4 \mathrm{GeV}$, the JeWEL+PYTHIA results agree with the data on a level of roughly $15 \%$ over about 6 decades and thus provide a realistic baseline for the $R_{\mathrm{AA}}$ determination. At LHC energies the hadron spectrum seems to be slightly softer in the simulation than in data. However, deviations of this size have only a very small effect on the nuclear modification factor. The uncertainty arising from this is much smaller than, for instance, the uncertainty arising from the choice of infrared regulator $\mu_{\mathbf{D}}$. 


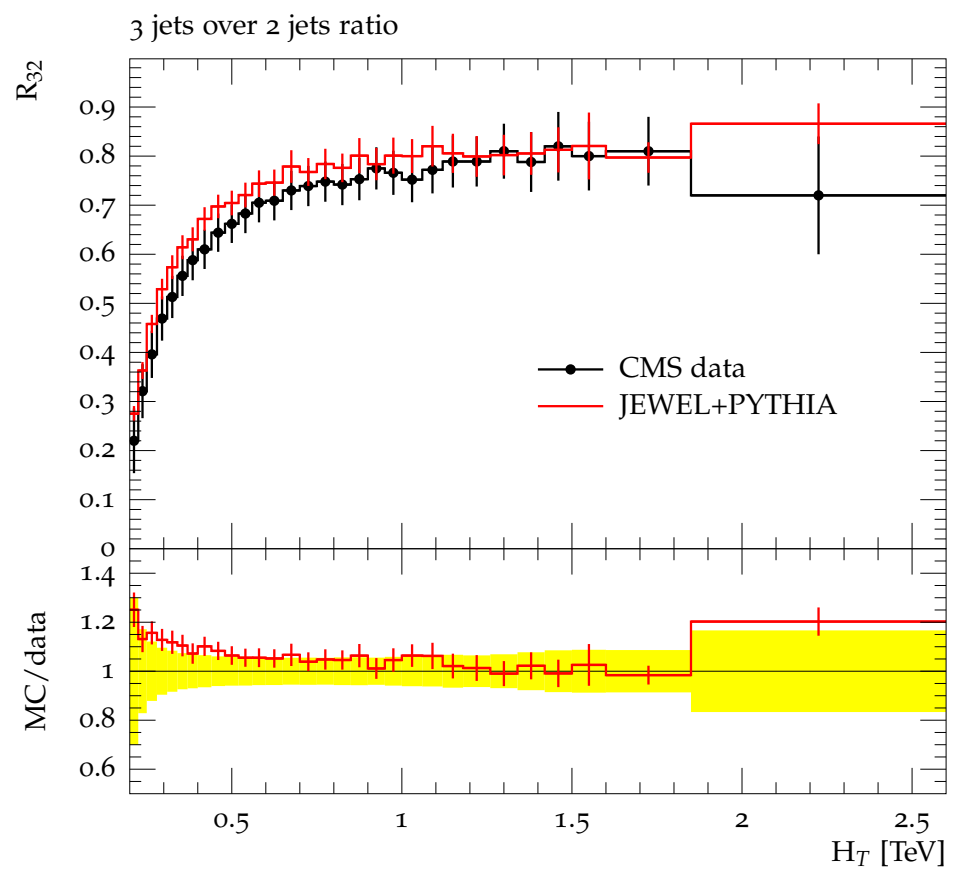

Figure 7. The ratio $R_{32}$ of the inclusive 3 -jet to the 2-jet cross section as a function of the scalar sum of the transverse momenta $H_{\mathrm{T}}$, the anti- $k_{\perp}$ jets in this analysis have $p_{\perp}>50 \mathrm{GeV}$ and $R=0.5[76]$.
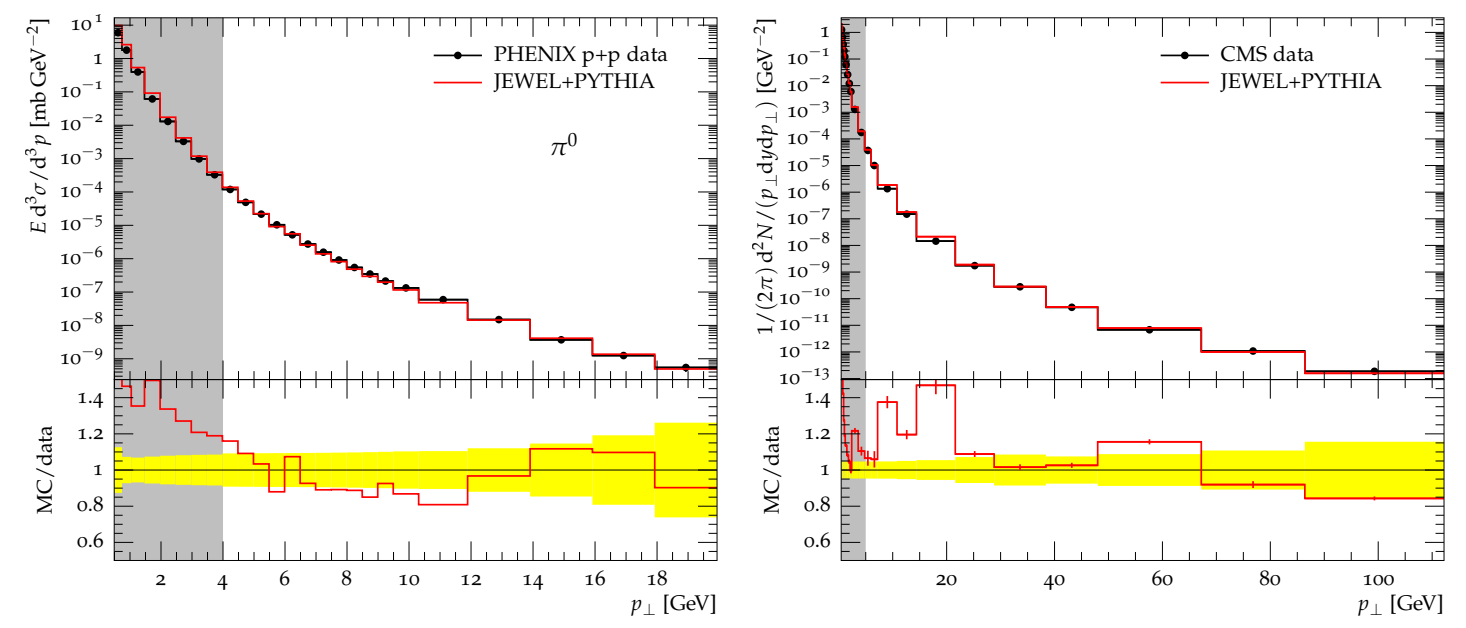

Figure 8. L.h.s.: neutral pion spectrum in $\mathrm{p}+\mathrm{p}$ collisions at $\sqrt{s}=200 \mathrm{GeV}$ simulated with Jewel + Pythia and compared to Phenix data [77]. R.h.s.: charged hadron spectrum in $\mathrm{p}+\mathrm{p}$ collisions at $\sqrt{s}=2.76 \mathrm{TeV}$ simulated with Jewel+PythiA and compared to CMS data [18].

\subsection{Hadron suppression}

To obtain a fair agreement with the measured nuclear modification factor at RHIC, JEWEL + PYTHIA requires an initial temperature of $T_{\mathrm{i}}=350 \mathrm{MeV}$ at initial proper time $\tau_{\mathrm{i}}=0.8 \mathrm{fm}$, see figure 9 . This is remarkably consistent with the input parameters in fluid dynamic simulations of heavy ion collisions [79-81]. We note that at high $p_{\perp}$, where the 


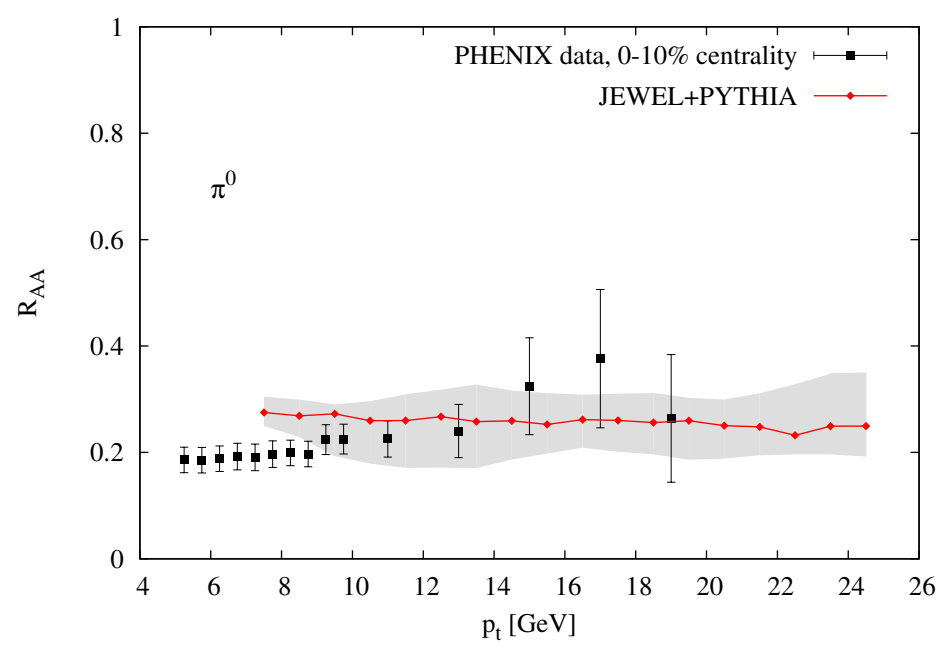

Figure 9. Nuclear modification factor for neutral pions in $\mathrm{Au}+\mathrm{Au}$ collisions at $\sqrt{s}=200 \mathrm{~A} \mathrm{GeV}$ in the $0-10 \%$ centrality class simulated with Jewel+Pythia and compared to PheniX data [78] (only systematic errors shown, statistical errors are smaller than the systematic ones everywhere). The grey band indicates a variation of the Debye mass by $\pm 10 \%$.

Monte Carlo results are reliable, they reproduce both the factor $\sim 5$ suppression and the approximately flat $p_{\perp}$-dependence seen in data. Varying the Debye mass by $\pm 10 \%$ has a significant effect on the overall suppression, indicated by the grey band, but hardly affects the shape. The dependence on the exact choice of the regulator is clearly sizeable. The pragmatic approach is to regard it as a parameter, fix it together with the medium parameters at one point (in this case the neutral pion suppression at RHIC) and use the same value for all calculations. But while this may be a practical working solution it does not address the underlying problem.

The charged hadron multiplicities measured in heavy ion collisions constrain the initial entropy density of the system, $s_{\mathrm{i}} \tau_{\mathrm{i}} \propto \mathrm{d} N / \mathrm{d} y, s_{\mathrm{i}} \propto \epsilon_{\mathrm{i}} / T_{\mathrm{i}} \propto T_{\mathrm{i}}^{3}$ and therefore allow to relate the initial temperatures at RHIC and at the LHC,

$$
T_{\mathrm{i}}^{\mathrm{LHC}}=T_{\mathrm{i}}^{\mathrm{RHIC}}\left(\frac{\tau_{\mathrm{i}}^{\mathrm{RHIC}}}{\tau_{\mathrm{i}}^{\mathrm{LHC}}} \frac{\mathrm{d} N /\left.\mathrm{d} y\right|_{\mathrm{LHC}}}{\mathrm{d} N /\left.\mathrm{d} y\right|_{\mathrm{RHIC}}}\right)^{1 / 3} .
$$

The observation of a factor 2.2 increase in the event multiplicity from RHIC to LHC is therefore consistent with an initial temperature $T_{\mathrm{i}}=530 \mathrm{MeV}$ at $\tau_{\mathrm{i}}=0.5 \mathrm{fm}$ at the LHC. There is some freedom in initialising the fluid dynamic evolution at the LHC at a different initial time $\tau_{\mathrm{i}}$, but this is numerically unimportant. At early times the parton shower is dominated by emissions at rather high scales initiated by the initial hard scattering, and this high virtuality protects the partons from medium-induced emissions and makes them insensitive to the medium at early times. Other observables might help to constrain the initial time further, but as JEWEL does not attempt to describe the entire nucleus-nucleus collision it does not have access to these and thus remains fairly insensitive to the initial time. Thus, the medium at the LHC is specified in terms of parameters fixed in figure 9 . As seen from figure 10, the calculation of JEWEL+PYTHIA then leads to a very good 


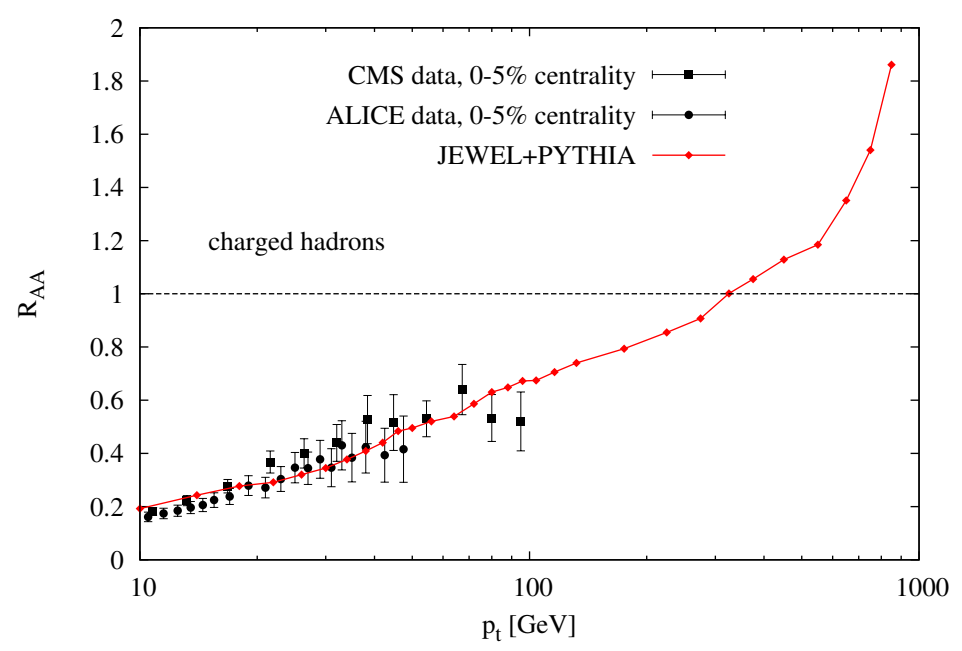

Figure 10. Nuclear modification factor for charged hadrons in $\mathrm{Pb}+\mathrm{Pb}$ collisions at $\sqrt{s}=$ $2.76 \mathrm{~A} \mathrm{TeV}$ in the $0-5 \%$ centrality class simulated with JEWEL+PYTHIA and compared to CMS [18] and Alice [16] data.

agreement with measurements of the nuclear modification factor at the LHC without any additional adjustments. This is a remarkable success of our model.

To understand the characteristically different $p_{\perp}$-dependencies of $R_{\mathrm{AA}}$ at RHIC and at the LHC, we have performed control simulations in which a LHC-like distribution of hard processes is fragmented in a RHIC-like medium, and vice versa (data not shown). This showed that the significant change in the slope of $R_{\mathrm{AA}}\left(p_{\perp}\right)$ from RHIC to the LHC can be attributed fully to the $\sqrt{s}$-dependence of the distribution of initial hard processes. We therefore conclude that a purely perturbative dynamics of parton energy loss supplemented by an arguably simple model of the medium whose characterisation matches physical expectations, can account for the main features of the measured nuclear modification factors, including the strength of the suppression pattern, and its $\sqrt{s}$ - and $p_{\perp}$-dependence.

At very large $p_{\perp}$ the nuclear modification factor continues to rise above unity. This is a purely kinematical effect that becomes visible at very large $p_{\perp}$ where the energy loss starts to vanish. The elastic scattering of energetic partons converts longitudinal into transverse momentum turning the $p_{\perp}$-spectrum harder. While this is a generic effect its size and turn-on point will to some degree depend on the medium model, as they are sensitive to the amount of scattering centres encountered in the forward direction. This effect would not be accessible in the standard eikonal treatment of parton energy loss that neglects the change in propagation direction of the projectile parton. We believe it is thus a generic testable prediction of our model.

\subsection{Modification of jets}

Analogously to the single-inclusive hadron suppression discussed in the previous section also the single-inclusive jet suppression has been measured by ALICE and ATLAS. In figures 11 and 12 the experimental results for different values of the jet radius are compared to Jewel+Pythia. The Alice results (which are relative to a pp baseline) and the Atlas 

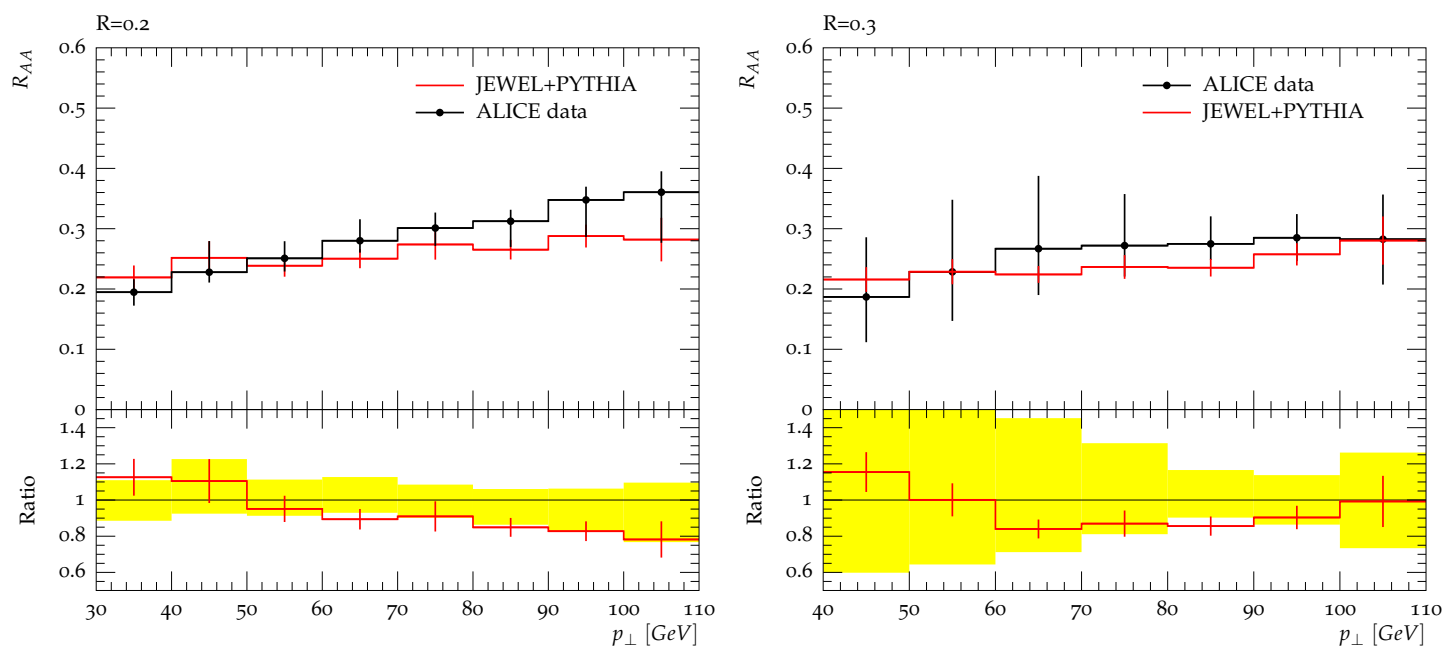

Figure 11. JeWEL + PythiA results for $R_{\mathrm{AA}}$ of jets in $\mathrm{Pb}+\mathrm{Pb}$ collisions at $\sqrt{s_{\mathrm{NN}}}=2.76 \mathrm{TeV}$ compared to ALICE data [21] for two values of the jet radius (correlated systematic errors not shown).
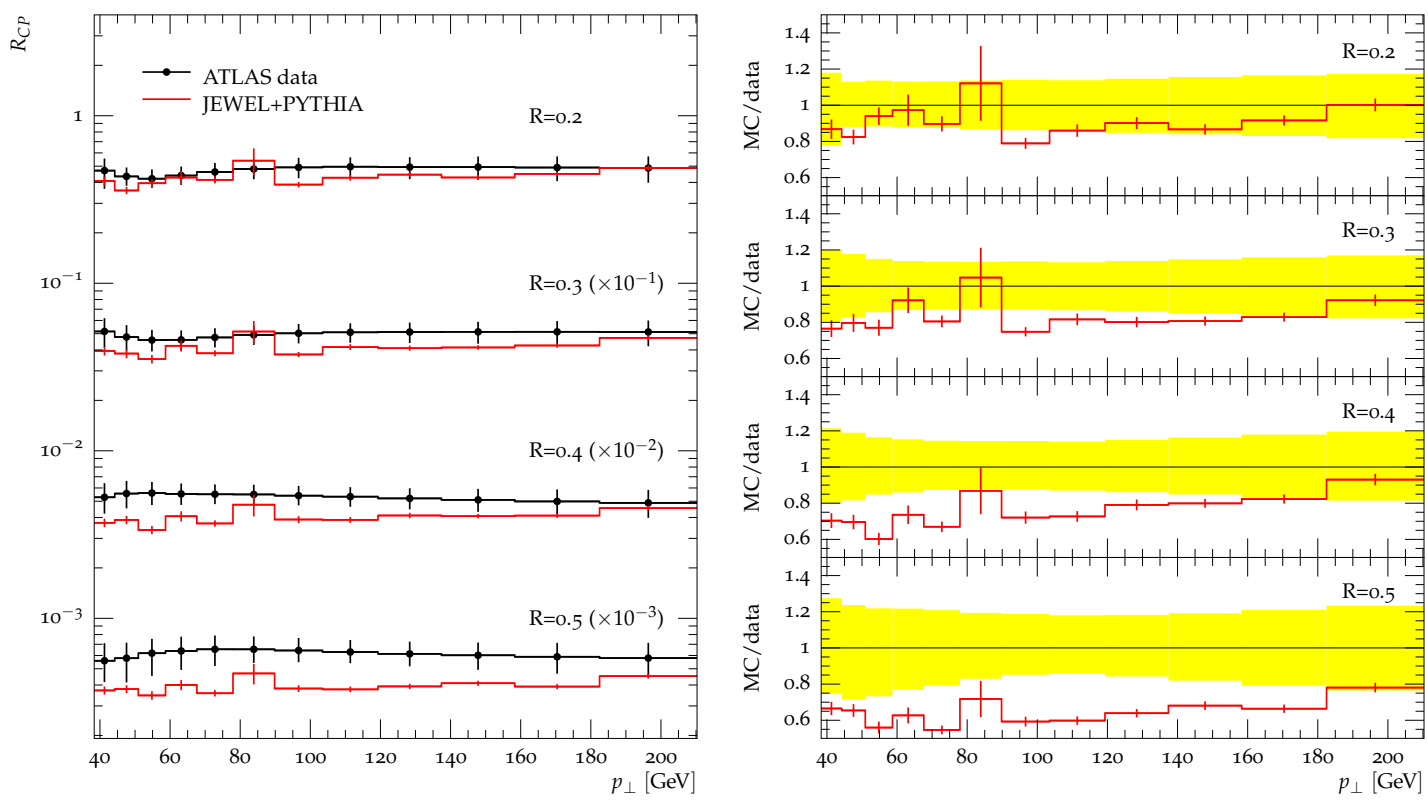

Figure 12. JeWEL + PythiA results for $R_{\mathrm{CP}}$ of jets in $\mathrm{Pb}+\mathrm{Pb}$ collisions at $\sqrt{s_{\mathrm{NN}}}=2.76 \mathrm{TeV}$ compared to ATLAS data [22] for different values of the jet radius. The ratio is taken between the $0-10 \%$ and the $60-80 \%$ centrality class.

results (which are relative to peripheral $\mathrm{Pb}+\mathrm{Pb}$ spectra) for small jet radii are very well reproduced, but there is some discrepancy in the ATLAS results for large jet radii. Also, in the $R=0.2$ measurement by ALICE, the $p_{\perp}$-dependence seems to be weaker in the MC than in the data. There is no indication of a significant rise of the jet suppression at large $p_{\perp}$ in the simulation, which shows that jets can behave differently from leading hadrons. The fact that the disagreement between JEWEL+PYTHIA and the ATLAS data increases with the jet radius $R$ could hint at an issue with the background subtraction. 

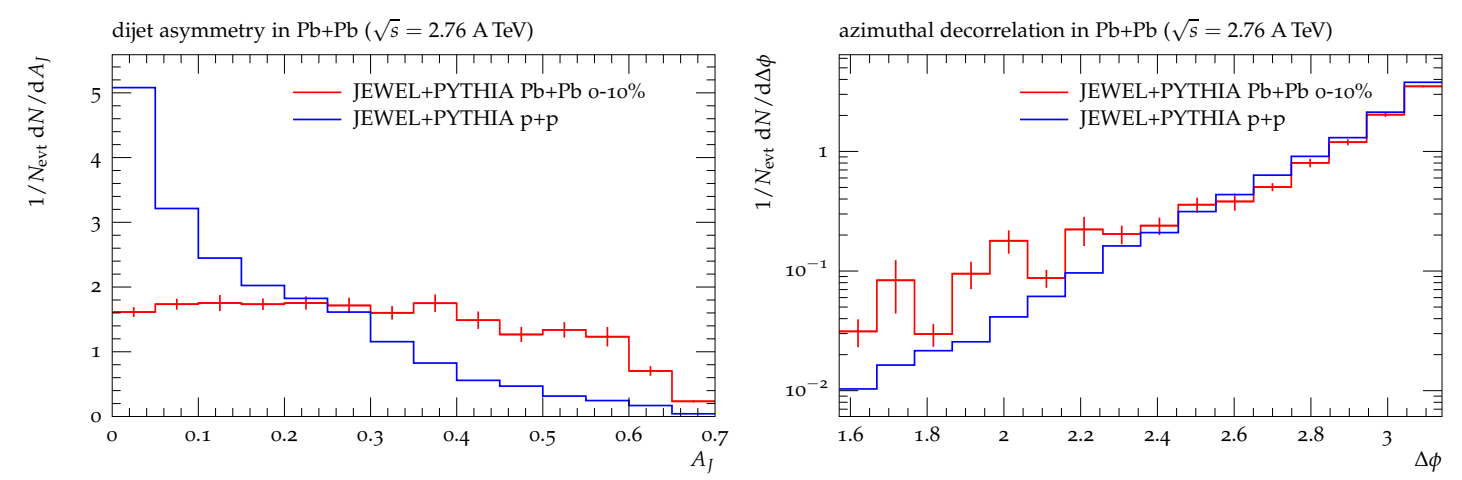

Figure 13. Di-jet asymmetry $A_{J}=\left(E_{\perp, 1}-E_{\perp, 2}\right) /\left(E_{\perp, 1}+E_{\perp, 2}\right)$ and azimuthal decorrelation in central $\mathrm{Pb}+\mathrm{Pb}$ and $\mathrm{p}+\mathrm{p}$ collisions at $\sqrt{s_{\mathrm{NN}}}=2.76 \mathrm{TeV}$, the cuts are the same as in the ATLAS analysis [19].

For the simulation of the single-inclusive hadron spectra the recoiling scattering centres are kept in the event and hadronised together with the jets. In the case of jet analyses, however, the recoils have to be taken out, since the experimental jet reconstruction involves the subtraction of background. These two procedures are not exactly identical for several reasons. Firstly, in JEWEL+PYTHIA the hadronic final state is not the incoherent sum of a jet component and a contribution from the recoils, due to the colour connections between the jet partons and the recoiling scattering centres. Secondly, in the experimental jet reconstruction procedure, only a background estimate based on the average activity in the event can be subtracted. While to some degree it is possible to deal with background fluctuations using unfolding techniques [82-84], the correlations between the jet and the background cannot be assessed in this way. There will thus always remain a residual uncertainty when comparing any MC results to jet data. Clearly, along the same line of reasoning, the background subtraction becomes more involved as the jet radius increases and the observation that the discrepancy between JEWEL+PYTHIA and data increases with jet radius may hint at a problem in this direction.

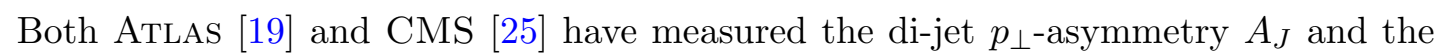
azimuthal decorrelation in central $\mathrm{Pb}+\mathrm{Pb}$ events. The JEwEL +PYTHIA results obtained with the ATLAS cuts are shown in figure 13. A direct quantitative comparison to either ATLAS or CMS data is not possible, as the data are not unfolded for the jet energy resolution, which is known to have a sizeable effect especially on the shape of the di-jet asymmetry. Qualitatively, however, the behaviour of the JEWEL+PYTHIA results resembles observations made in the data: There is a significant broadening of the $A_{J}$ distribution in central $\mathrm{Pb}+\mathrm{Pb}$ events as compared to the $\mathrm{p}+\mathrm{p}$ baseline. The azimuthal decorrelation, on the other hand, hardly changes; there is only a mild increase at separations $\Delta \phi \sim \pi / 2$.

The charged particle fragmentation function in jets in central $\mathrm{Pb}+\mathrm{Pb}$ collisions as a function of the momentum fraction $z$ and relative $p_{\perp}$ in central and peripheral events are shown in figure 14 and figure 15, respectively. Except for the region of very small $z$ or $p_{\perp}$, which is expected to be particularly sensitive to background modelling and subtraction, the agreement between the JEWEL+PYTHIA results and the data is very reasonable given 

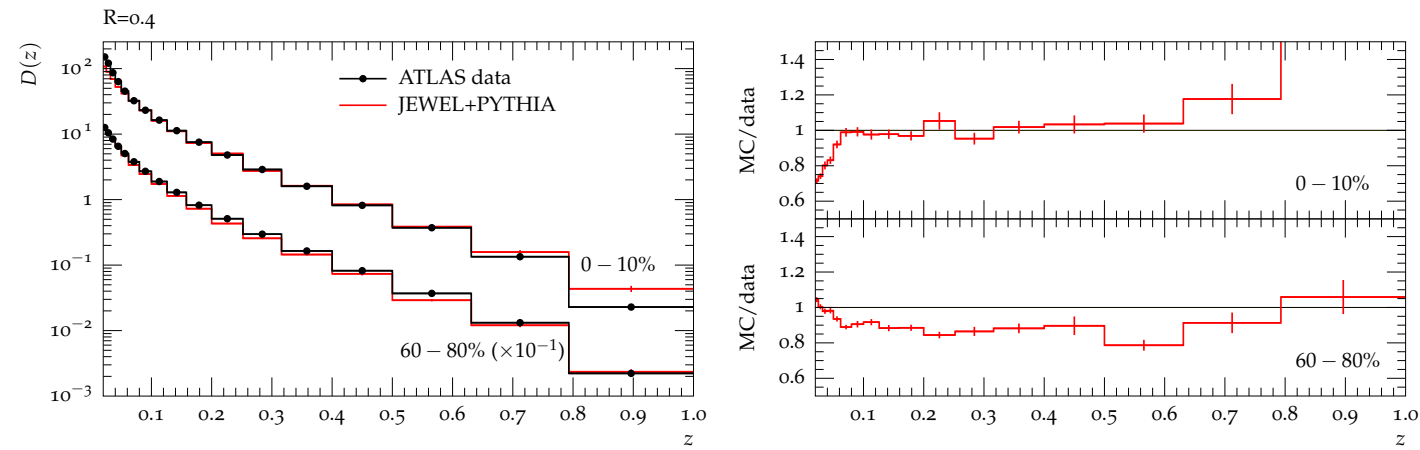

Figure 14. JeweL + PythiA results for the fragmentation function $D(z)$ in peripheral and central $\mathrm{Pb}+\mathrm{Pb}$ events compared to ATLAS data [26] (data points read off the plots, no errors shown).
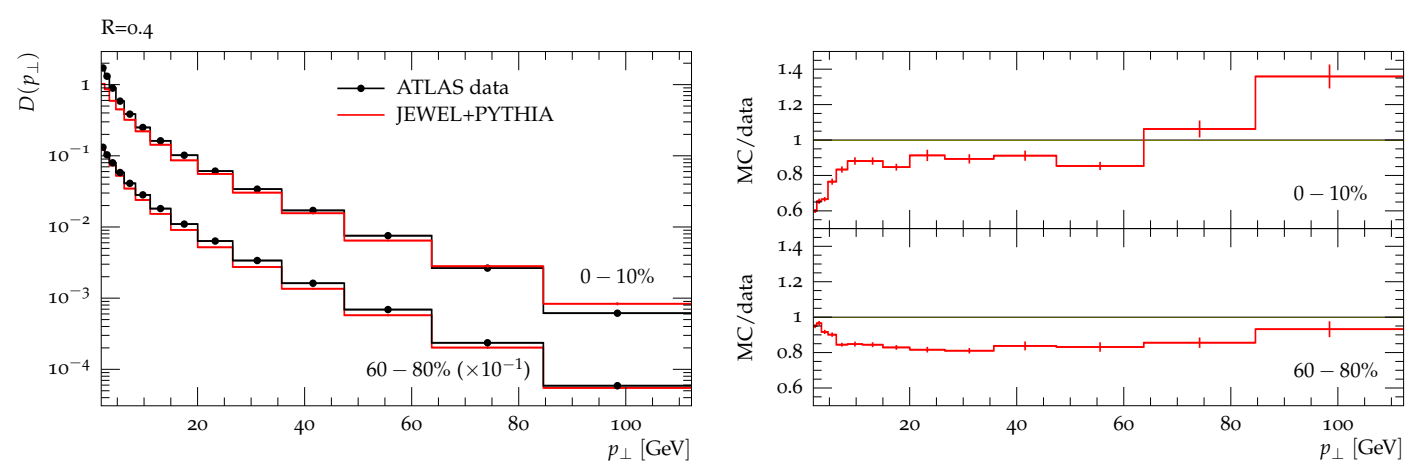

Figure 15. JeWEL + PythiA results for the fragmentation function $D\left(p_{\perp}\right)$ in peripheral and central $\mathrm{Pb}+\mathrm{Pb}$ events compared to ATLAS data [26] (data points read off the plots, no errors shown).
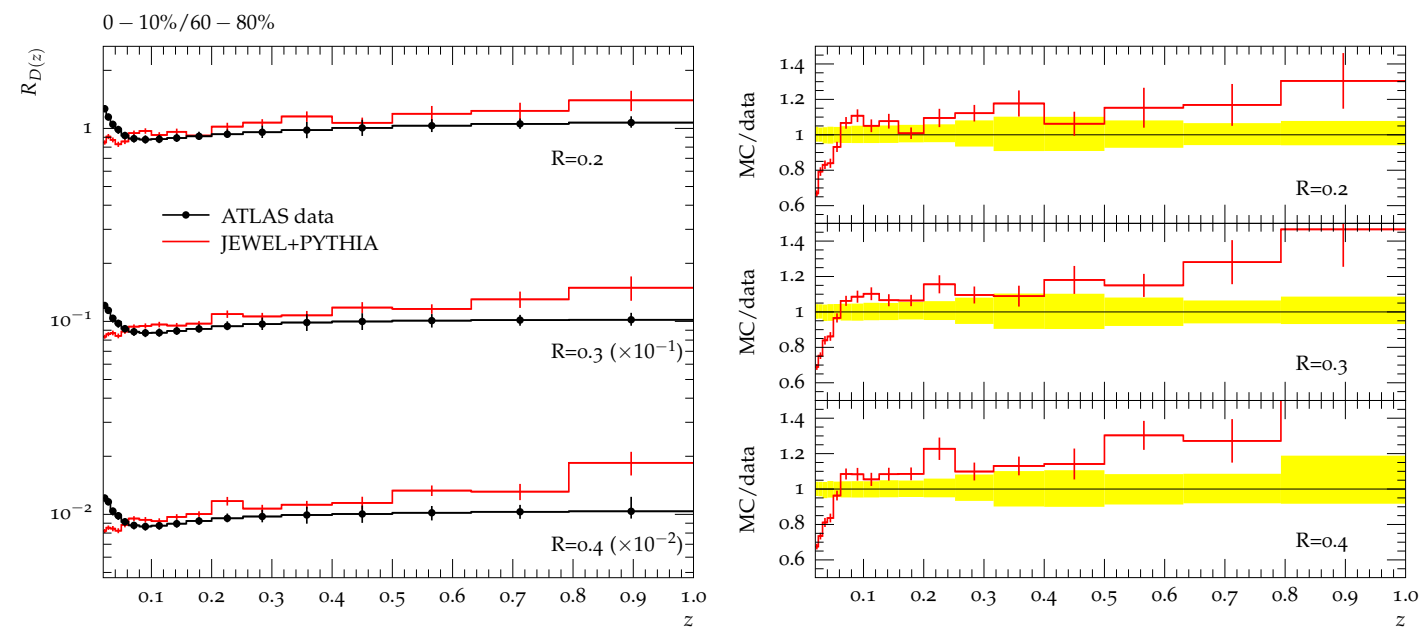

Figure 16. Jewel+Pythia results for the ratios of the fragmentation functions $D(z)$ between central and peripheral $\mathrm{Pb}+\mathrm{Pb}$ events compared to ATLAS data [26] (data points read off the plots, only maximum of statistical and systematic errors shown).

that for this measurement the background subtraction is more involved than for jets. It remains unclear, however, whether the observation, that the fragmentation function in $\mathrm{p}+\mathrm{p}$ at $\sqrt{s}=7 \mathrm{TeV}$ seems to be too soft, indicates that in $\mathrm{Pb}+\mathrm{Pb}$ it is actually too hard. The 

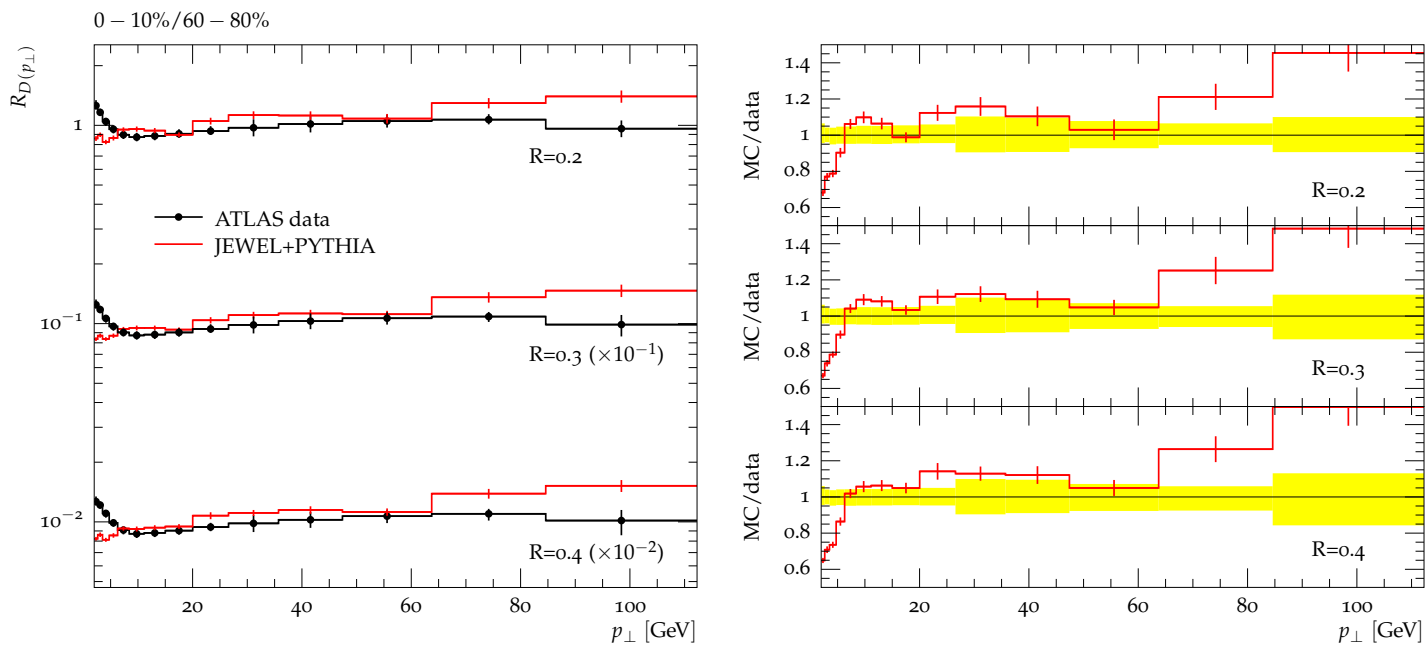

Figure 17. JEWEL+PyTHIA results for the ratios of the fragmentation functions $D\left(p_{\perp}\right)$ between central and peripheral $\mathrm{Pb}+\mathrm{Pb}$ events compared to ATLAS data [26] (data points read off the plots, only maximum of statistical and systematic errors shown).
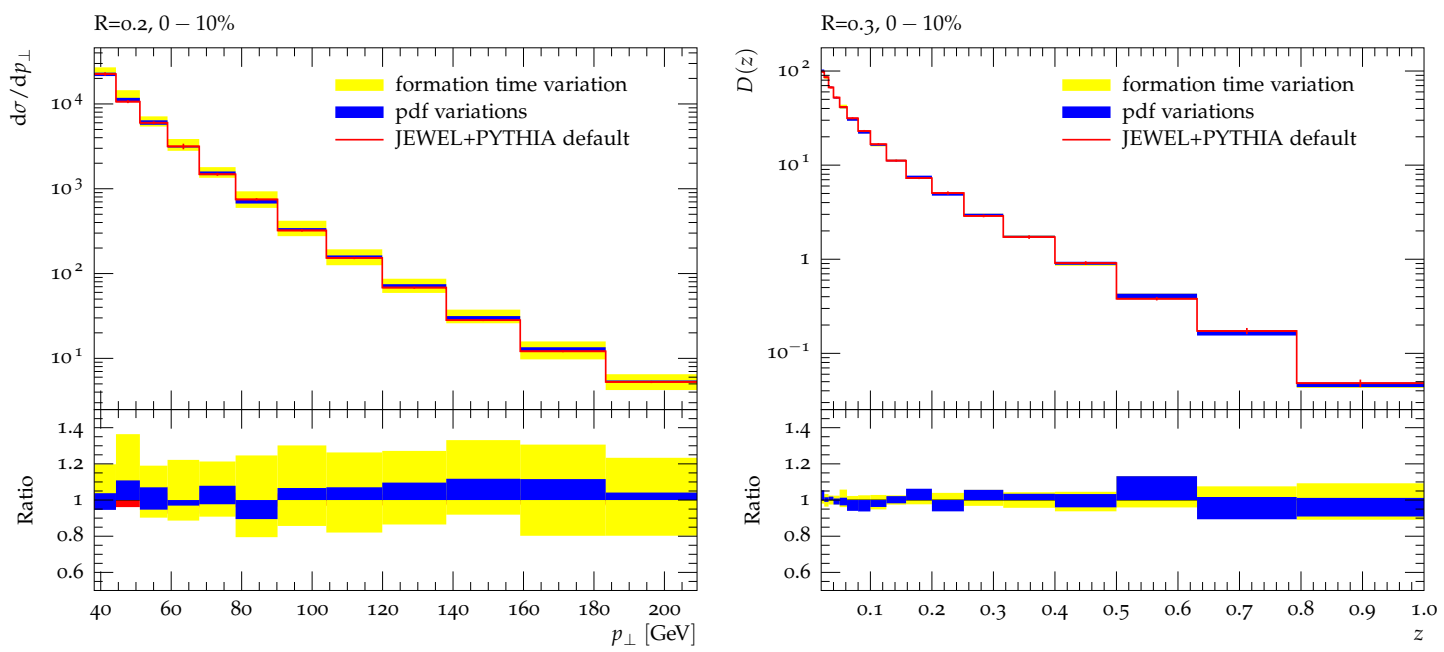

Figure 18. Uncertainties due to varying the formation time (yellow band) and the PDF (blue band) on the inclusive jet spectrum (l.h.s.) and the fragmentation function (r.h.s.) in central $\mathrm{Pb}+\mathrm{Pb}$ collisions. The statistical uncertainty on the default set-up is shown as error bars in the upper panel and as the red band in the ratio plots.

ratio of the fragmentation functions in central and peripheral events shown in figure 16 and figure 17 are less sensitive to such effects. Again, the agreement between data and MC improves for smaller values of the jet radius. In JEWEL+PYTHIA the fragmentation function tends to become harder in more central events. This can easily be understood since the fragmentation of the hard core remains unaltered by the presence of the medium while the jet energy gets degraded. 

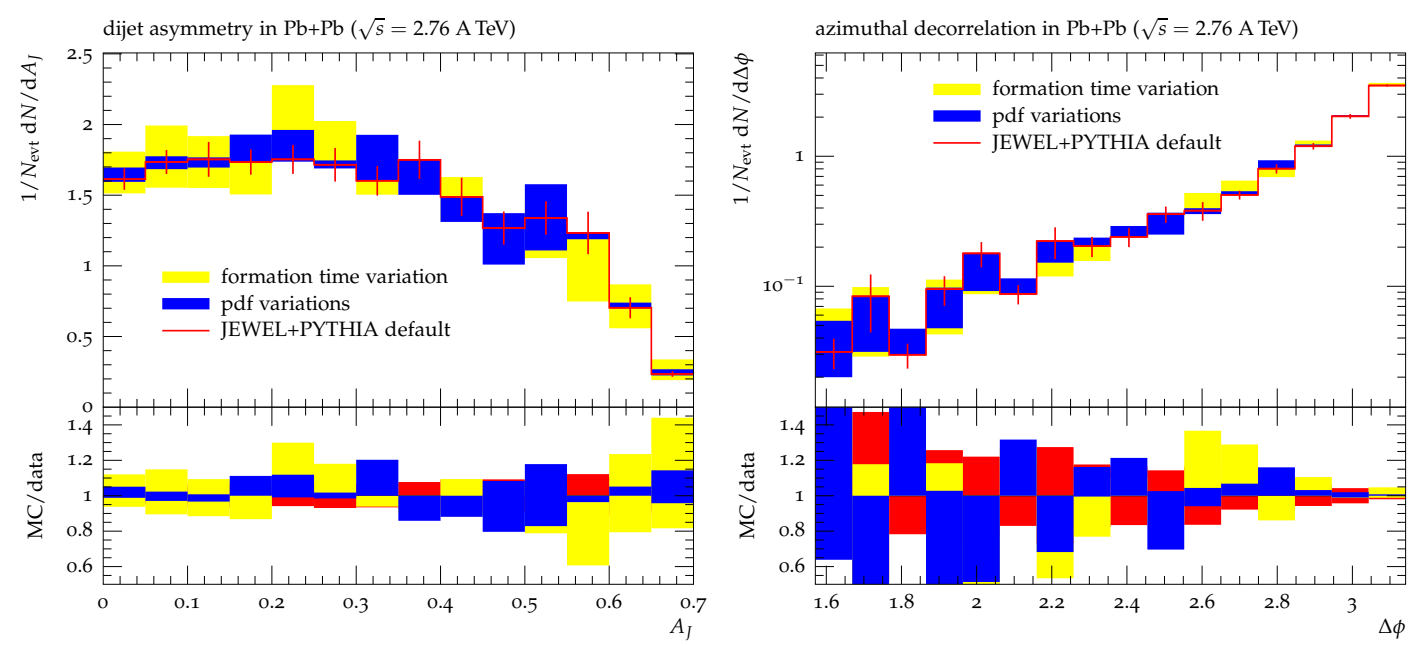

Figure 19. Uncertainties due to varying the formation time (yellow band) and the PDF (blue band) on the di-jet asymmetry (l.h.s.) and the azimuthal decorrelation (r.h.s.) in central $\mathrm{Pb}+\mathrm{Pb}$ collisions. The statistical uncertainty on the default set-up is shown as error bars in the upper panel and as the red band in the ratio plots.

\subsection{Uncertainties}

Uncertainties related to the basic assumptions and other aspects of the calculation (e.g. PDF uncertainties) are to some extent unavoidable, but can be quantified in our model. While a complete assessment of all possible uncertainties certainly is beyond the scope of this publication, we identify and discuss the most important sources of uncertainties and their respective sizes.

Apart from the model of the medium the framework is essentially free of parameters. There are choices to be made concerning the infrared regulator of the matrix elements and the formation time, but once they are fixed they should not be changed. The infrared cut-off of the parton shower and the parameters of the hadronisation model can be tuned to LEP data and are not changed when going to nucleus-nucleus collisions. There is thus only very little room for adjustments and tuning.

The effect of varying the infrared regulator $\mu_{\mathrm{D}}$, which presents the largest uncertainty, was already discussed in the context of the single-inclusive hadron suppression. In this section we show the effect of varying the formation time up and down by a factor 2 and using different PDFs (Cteq6L1[64]+EPs09Lo[66], which is the default, Cteq6L1 without nuclear modifiactions and Mstw08Lo[85]+Eps09LO). Figure 18 shows the effect on the inclusive jet spectrum and the fragmentation function and figure 19 the di-jet asymmetry and azimuthal decorrelation. Varying the formation times leads to a $\sim 20 \%$ variation of the jet rate in central $\mathrm{Pb}+\mathrm{Pb}$ collisions and a smaller but visible change in the di-jet asymmetry and azimuthal decorrelation while the fragmentation function is fairly insensitive. The uncertainty related to the choice of PDF is smaller and of the same size as the current statistical uncertainty on the MC results.

In a similar way practically all aspects of the simulation can be varied and the uncertainties estimated. We find that the largest sources of uncertainties are the choice of the infrared regulator and to a lesser degree the formation time. 


\section{Conclusions}

In this paper we report on the development of a novel description of jet quenching and its implementation into the Monte Carlo generator JEwEL. The paradigm underlying the construction of our model is the language given by perturbative QCD, with the aim to enable a quantitative discussion of effects beyond it. In this context, hard interactions of a jet with the medium resolve its constituents as quasi-free partons and should be described in perturbation theory. ${ }^{7}$ In reinterpreting induced radiation as regular, although infra-redregulated, $2 \rightarrow 2$ parton scatterings, supplemented with a parton shower we arrive at a framework whose dynamics is entirely based on standard perturbative technology also used in the simulation of $p+p$ collisions and which is minimal in its assumptions. It is formulated entirely in general kinematics ${ }^{8}$ and thus overcomes limitations of previous approaches which were entirely based on results obtained in eikonal or close-to-eikonal kinematics [48]. Consequently, there is no distinction between elastic and inelastic scattering. Rather, both are possible outcomes of the same process and the parton shower cut-off defines the separation between the two. Also the distinction between vacuum and medium-induced radiation has become obsolete, as they are fundamentally the same. The interplay between all sources of radiation is governed by the formation times and generally a certain emission cannot be classified as vacuum-like or medium-induced in a meaningful way.

The emerging framework is well constrained by data from elementary reactions and does not leave much room for tuning. Apart from the model of the medium there is only a single quantity with a significant uncertainty that should perhaps be regarded as a parameter, namely the infrared regulator of the matrix elements. All uncertainties related to assumptions or other aspects of the calculations can be quantified, examples for the numerically most important uncertainties have been presented here, but a complete characterisation of all possible uncertainties is beyond the scope of this publication.

Our new framework does not make any assumptions about the medium other than that it is partonic. The phase space distribution does not matter and in principle any distribution can be interfaced. The results presented here were obtained with a simple Bjorken type model. Nevertheless, after fixing the parameters with RHIC data, the agreement with very different jet and leading hadron measurements at the LHC is very reassuring. This leads us to conclude that our calculation, although certain aspects are simplified, captures the relevant physics underlying jet quenching. At the same time the overall good description of data lets us speculate in how far more complicated medium models may obfuscate simple physical realities. This clearly is a question that could only be answered seriously by interfacing more medium models and cataloguing the results obtained with them.

However, we would like to stress that for some observables there appear to be unresolved and potentially unresolvalve issues with the subtraction of backgrounds, where the

\footnotetext{
${ }^{7}$ In general, the scale above which the components of a final state parton shower can resolve partonic constituents of the medium as quasi-free partons depends on properties of the medium [86]. Comparing a perturbative formulation of jet quenching to data can thus give access to the nature of medium constituents.

${ }^{8}$ Although the present code goes far beyond the eikonal kinematics, it is clear how to recover this limit of the full emission pattern encoded here.
} 
procedure in the analysis of $\mathrm{MC}$ events differs from the experimental one and may lead to discrepancies. The observation that generally the agreement between $\mathrm{MC}$ and data gets worse for larger jet radii points in this direction. Also, it should be noted that there is a class of measurements that are sensitive to the back-reaction of the jet on the medium and that therefore our calculation cannot be expected to describe. This problem could only be overcome by trying to extend our model to the fully inclusive treatment of the full collision - something clearly beyond the scope of this paper.

Apart from the obvious option of testing different models for the medium we have hinted at various other ways of how our calculation can systematically be improved using a well-understood perturbative language.

Open Access. This article is distributed under the terms of the Creative Commons Attribution License which permits any use, distribution and reproduction in any medium, provided the original author(s) and source are credited.

\section{References}

[1] T. Gleisberg et al., Event generation with SHERPA 1.1, JHEP 02 (2009) 007 [arXiv: 0811.4622] [INSPIRE].

[2] T. Sjöstrand, S. Mrenna and P.Z. Skands, A brief introduction to PYTHIA 8.1, Comput. Phys. Commun. 178 (2008) 852 [arXiv:0710.3820] [InSPIRE].

[3] M. Bahr et al., HERWIG++ physics and manual, Eur. Phys. J. C 58 (2008) 639 [arXiv:0803.0883] [INSPIRE].

[4] A. Buckley et al., General-purpose event generators for LHC physics, Phys. Rept. 504 (2011) 145 [arXiv: 1101.2599] [INSPIRE].

[5] M. Bengtsson and T. Sjöstrand, A comparative study of coherent and noncoherent parton shower evolution, Nucl. Phys. B 289 (1987) 810 [INSPIRE].

[6] G. Marchesini and B. Webber, Monte Carlo simulation of general hard processes with coherent QCD radiation, Nucl. Phys. B 310 (1988) 461 [inSPIRE].

[7] L. Lönnblad, ARIADNE version 4: a program for simulation of QCD cascades implementing the color dipole model, Comput. Phys. Commun. 71 (1992) 15 [INSPIRE].

[8] S. Gieseke, P. Stephens and B. Webber, New formalism for QCD parton showers, JHEP 12 (2003) 045 [hep-ph/0310083] [INSPIRE].

[9] Z. Nagy and D.E. Soper, Matching parton showers to NLO computations, JHEP 10 (2005) 024 [hep-ph/0503053] [INSPIRE].

[10] W.T. Giele, D.A. Kosower and P.Z. Skands, A simple shower and matching algorithm, Phys. Rev. D 78 (2008) 014026 [arXiv:0707.3652] [INSPIRE].

[11] S. Schumann and F. Krauss, A parton shower algorithm based on Catani-Seymour dipole factorisation, JHEP 03 (2008) 038 [arXiv:0709.1027] [INSPIRE].

[12] M. Dinsdale, M. Ternick and S. Weinzierl, Parton showers from the dipole formalism, Phys. Rev. D 76 (2007) 094003 [arXiv: 0709.1026] [InSPIRE].

[13] J.-C. Winter and F. Krauss, Initial-state showering based on colour dipoles connected to incoming parton lines, JHEP 07 (2008) 040 [arXiv:0712.3913] [INSPIRE]. 
[14] PHENIX collaboration, A. Adare et al., Suppression pattern of neutral pions at high transverse momentum in $A u+A u$ collisions at $\sqrt{s_{N N}}=200 \mathrm{GeV}$ and constraints on medium transport coefficients, Phys. Rev. Lett. 101 (2008) 232301 [arXiv:0801.4020] [INSPIRE].

[15] STAR collaboration, B. Abelev et al., Energy dependence of $\pi^{ \pm}, p$ and $\bar{p}$ transverse momentum spectra for Au+Au collisions at $\sqrt{s_{N N}}=62.4$ and $200 \mathrm{GeV}$, Phys. Lett. B 655 (2007) 104 [nucl-ex/0703040] [INSPIRE].

[16] ALICE collaboration, Centrality dependence of charged particle production at large transverse momentum in $\mathrm{Pb}-\mathrm{Pb}$ collisions at $\sqrt{s_{\mathrm{NN}}}=2.76 \mathrm{TeV}$, Phys. Lett. B 720 (2012) 52 [arXiv: 1208.2711] [INSPIRE].

[17] ATLAS collaboration, Measurement of the charged particle spectra in $\mathrm{PbPb}$ collisions at $s q n=2.76 \mathrm{TeV}$ with the ATLAS detector at the LHC, ATLAS-CONF-2012-120 (2012).

[18] CMS collaboration, , Study of high- $p_{T}$ charged particle suppression in PbPb compared to $p p$ collisions at $\sqrt{s_{N N}}=2.76 \mathrm{TeV}$, Eur. Phys. J. C 72 (2012) 1945 [arXiv:1202.2554] [INSPIRE].

[19] ATLAS collaboration, Observation of a centrality-dependent dijet asymmetry in lead-lead collisions at $\sqrt{s_{N N}}=2.77 \mathrm{TeV}$ with the ATLAS detector at the LHC, Phys. Rev. Lett. 105 (2010) 252303 [arXiv: 1011.6182] [INSPIRE].

[20] CMS collaboration, Observation and studies of jet quenching in PbPb collisions at nucleon-nucleon center-of-mass energy $=2.76$ TeV, Phys. Rev. C 84 (2011) 024906 [arXiv: 1102.1957] [INSPIRE].

[21] ALICE collaboration, Measurement of jet spectra in Pb-Pb collisions at $\sqrt{s_{N N}}=2.76 \mathrm{TeV}$ with the ALICE detector at the LHC, arXiv:1208.6169 [INSPIRE].

[22] ATLAS collaboration, Measurement of the jet radius and transverse momentum dependence of inclusive jet suppression in lead-lead collisions at $\sqrt{s_{N N}}=2.76 \mathrm{TeV}$ with the ATLAS detector, Phys. Lett. B 719 (2013) 220 [arXiv:1208.1967] [InSPIRE].

[23] M. Cacciari, G.P. Salam and G. Soyez, FastJet user manual, Eur. Phys. J. C 72 (2012) 1896 [arXiv:1111.6097] [INSPIRE].

[24] M. Cacciari, G.P. Salam and G. Soyez, The catchment area of jets, JHEP 04 (2008) 005 [arXiv:0802.1188] [INSPIRE].

[25] CMS collaboration, Measurement of jet fragmentation into charged particles in pp and $\mathrm{PbPb}$ collisions at $\sqrt{s_{N N}}=2.76 \mathrm{TeV}$, JHEP 10 (2012) 087 [arXiv:1205.5872] [INSPIRE].

[26] ATLAS collaboration, Measurement of inclusive jet charged particle fragmentation functions in $\mathrm{Pb}+\mathrm{Pb}$ collisions at $\sqrt{s_{n n}}=2.76 \mathrm{TeV}$ with the ATLAS detector, ATLAS-CONF-2012-115 (2012).

[27] J.D. Bjorken, Energy loss of energetic partons in quark-gluon plasma: possible extinction of high $p_{t}$ jets in hadron-hadron collisions, FERMILAB-PUB-82-059-THY (1982) [INSPIRE].

[28] E. Braaten and M.H. Thoma, Energy loss of a heavy fermion in a hot plasma, Phys. Rev. D 44 (1991) 1298 [INSPIRE].

[29] M. Gyulassy and X.-n. Wang, Multiple collisions and induced gluon Bremsstrahlung in QCD, Nucl. Phys. B 420 (1994) 583 [nucl-th/9306003] [INSPIRE].

[30] D. d'Enterria, Jet quenching, arXiv:0902.2011 [INSPIRE].

[31] U.A. Wiedemann, Jet quenching in heavy ion collisions, arXiv:0908.2306 [INSPIRE]. 
[32] T. Renk, Modelling jet quenching, arXiv:1207.4885 [INSPIRE].

[33] J. Casalderrey-Solana and A. Milov, High-p $p_{T}$ and Jets. A summary of results from quark matter 2012, arXiv:1210.8271 [INSPIRE].

[34] R. Baier, Y.L. Dokshitzer, A.H. Mueller, S. Peigne and D. Schiff, Radiative energy loss of high-energy quarks and gluons in a finite volume quark-gluon plasma, Nucl. Phys. B 483 (1997) 291 [hep-ph/9607355] [INSPIRE].

[35] B.G. Zakharov, Radiative energy loss of high-energy quarks in finite size nuclear matter and quark-gluon plasma, JETP Lett. 65 (1997) 615 [hep-ph/9704255] [INSPIRE].

[36] U.A. Wiedemann, Gluon radiation off hard quarks in a nuclear environment: Opacity expansion, Nucl. Phys. B 588 (2000) 303 [hep-ph/0005129] [INSPIRE].

[37] M. Gyulassy, P. Levai and I. Vitev, Reaction operator approach to nonAbelian energy loss, Nucl. Phys. B 594 (2001) 371 [nucl-th/0006010] [INSPIRE].

[38] X.-N. Wang and X.-f. Guo, Multiple parton scattering in nuclei: Parton energy loss, Nucl. Phys. A 696 (2001) 788 [hep-ph/0102230] [INSPIRE].

[39] P.B. Arnold, G.D. Moore and L.G. Yaffe, Photon and gluon emission in relativistic plasmas, JHEP 06 (2002) 030 [hep-ph/0204343] [INSPIRE].

[40] X.-F. Chen, T. Hirano, E. Wang, X.-N. Wang and H. Zhang, Suppression of high $p_{T}$ hadrons in Pb+Pb collisions at LHC, Phys. Rev. C 84 (2011) 034902 [arXiv:1102.5614] [InSPIRE].

[41] W. Horowitz and M. Gyulassy, Quenching and tomography from RHIC to LHC, J. Phys. G 38 (2011) 124114 [arXiv:1107.2136] [INSPIRE].

[42] A. Majumder and C. Shen, Suppression of the high $p_{T}$ charged hadron $R_{A A}$ at the LHC, Phys. Rev. Lett. 109 (2012) 202301 [arXiv:1103.0809] [INSPIRE].

[43] B. Zakharov, Jet quenching from RHIC to LHC, arXiv:1105.0191 [INSPIRE].

[44] Y. Mehtar-Tani, C.A. Salgado and K. Tywoniuk, The radiation pattern of a QCD antenna in a dense medium, JHEP 10 (2012) 197 [arXiv:1205.5739] [INSPIRE].

[45] J. Casalderrey-Solana and E. Iancu, Interference effects in medium-induced gluon radiation, JHEP 08 (2011) 015 [arXiv: 1105.1760] [INSPIRE].

[46] A. Beraudo, J.G. Milhano and U.A. Wiedemann, The contribution of medium-modified color flow to jet quenching, JHEP 07 (2012) 144 [arXiv:1204.4342] [INSPIRE].

[47] K.C. Zapp, J. Stachel and U.A. Wiedemann, A local Monte Carlo framework for coherent QCD parton energy loss, JHEP 07 (2011) 118 [arXiv:1103.6252] [INSPIRE].

[48] N. Armesto et al., Comparison of jet quenching formalisms for a quark-gluon plasma 'brick', Phys. Rev. C 86 (2012) 064904 [arXiv:1106.1106] [INSPIRE].

[49] W.-T. Deng, X.-N. Wang and R. Xu, Hadron production in $p+p, p+P b$ and $\mathrm{Pb}+\mathrm{Pb}$ collisions with the HIJING 2.0 model at energies available at the CERN Large Hadron Collider, Phys. Rev. C 83 (2011) 014915 [arXiv: 1008.1841] [InSPIRE].

[50] I. Lokhtin et al., Heavy ion event generator HYDJET++ (HYDrodynamics plus JETs), Comput. Phys. Commun. 180 (2009) 779 [arXiv:0809.2708] [INSPIRE].

[51] K. Zapp, G. Ingelman, J. Rathsman, J. Stachel and U.A. Wiedemann, A Monte Carlo model for 'jet quenching', Eur. Phys. J. C 60 (2009) 617 [arXiv:0804.3568] [INSPIRE]. 
[52] N. Armesto, L. Cunqueiro and C.A. Salgado, Q-PYTHIA: a medium-modified implementation of final state radiation, Eur. Phys. J. C 63 (2009) 679 [arXiv:0907.1014] [INSPIRE].

[53] N. Armesto, G. Corcella, L. Cunqueiro and C.A. Salgado, Angular-ordered parton showers with medium-modified splitting functions, JHEP 11 (2009) 122 [arXiv:0909.5118] [INSPIRE].

[54] T. Renk, A comparison study of medium-modified QCD shower evolution scenarios, Phys. Rev. C 79 (2009) 054906 [arXiv:0901.2818] [inSPIRE].

[55] B. Schenke, C. Gale and S. Jeon, MARTINI: an event generator for relativistic heavy-ion collisions, Phys. Rev. C 80 (2009) 054913 [arXiv:0909.2037] [INSPIRE].

[56] C. Coleman-Smith and B. Müller, What can we learn from dijets? A systematic study with $V N I / B M S$, arXiv:1210.3377 [INSPIRE].

[57] J. Casalderrey-Solana, H. Liu, D. Mateos, K. Rajagopal and U.A. Wiedemann, Gauge/string duality, hot QCD and heavy ion collisions, arXiv:1101.0618 [INSPIRE].

[58] G. Gustafson and U. Pettersson, Dipole formulation of QCD cascades, Nucl. Phys. B 306 (1988) 746 [INSPIRE].

[59] Y.L. Dokshitzer, V.A. Khoze, A.H. Mueller and S. Troian, Basics of perturbative QCD, Atlantica Séguier Frontières, France (1991).

[60] R.K. Ellis, W. J. Stirling and B. Webber, QCD and collider physics, Cambridge Monographs on Particle Physics, Nuclear Physics and Cosmology volume 8, Cambridge University Press, Cambridge U.K. (1996).

[61] S. Catani, F. Krauss, R. Kuhn and B. Webber, QCD matrix elements + parton showers, JHEP 11 (2001) 063 [hep-ph/0109231] [INSPIRE].

[62] M.L. Mangano, M. Moretti, F. Piccinini and M. Treccani, Matching matrix elements and shower evolution for top-quark production in hadronic collisions, JHEP 01 (2007) 013 [hep-ph/0611129] [INSPIRE].

[63] T. Sjöstrand, S. Mrenna and P.Z. Skands, PYTHIA 6.4 physics and manual, JHEP 05 (2006) 026 [hep-ph/0603175] [INSPIRE].

[64] J. Pumplin et al., New generation of parton distributions with uncertainties from global QCD analysis, JHEP 07 (2002) 012 [hep-ph/0201195] [INSPIRE].

[65] M. Whalley, D. Bourilkov and R. Group, The Les Houches accord PDFs (LHAPDF) and LHAGLUE, hep-ph/0508110 [INSPIRE].

[66] K. Eskola, H. Paukkunen and C. Salgado, EPSO9: a new generation of NLO and LO nuclear parton distribution functions, JHEP 04 (2009) 065 [arXiv: 0902 .4154] [INSPIRE].

[67] J.D. Bjorken, Highly relativistic nucleus-nucleus collisions: the central rapidity region, Phys. Rev. D 27 (1983) 140 [INSPIRE].

[68] K. Zapp, G. Ingelman, J. Rathsman and J. Stachel, Jet quenching from soft QCD scattering in the quark-gluon plasma, Phys. Lett. B 637 (2006) 179 [hep-ph/0512300] [INSPIRE].

[69] K.J. Eskola, K. Kajantie and J. Lindfors, Quark and gluon production in high-energy nucleus-nucleus collisions, Nucl. Phys. B 323 (1989) 37 [INSPIRE].

[70] A. Buckley et al., Rivet user manual, arXiv:1003.0694 [INSPIRE]. 
[71] ALEPH collaboration, R. Barate et al., Studies of quantum chromodynamics with the ALEPH detector, Phys. Rept. 294 (1998) 1 [INSPIRE].

[72] DELPHI collaboration, P. Abreu et al., Tuning and test of fragmentation models based on identified particles and precision event shape data, Z. Phys. C 73 (1996) 11.

[73] ATLAS collaboration, Properties of jets measured from tracks in proton-proton collisions at center-of-mass energy $\sqrt{s}=7$ TeV with the ATLAS detector, Phys. Rev. D 84 (2011) 054001 [arXiv:1107.3311] [INSPIRE].

[74] ATLAS collaboration, Study of jet shapes in inclusive jet production in pp collisions at $\sqrt{s}=7$ TeV using the ATLAS detector, Phys. Rev. D 83 (2011) 052003 [arXiv:1101.0070] [INSPIRE].

[75] ATLAS collaboration, Measurement of dijet azimuthal decorrelations in pp collisions at $\sqrt{s}=7$ TeV, Phys. Rev. Lett. 106 (2011) 172002 [arXiv:1102.2696] [INSPIRE].

[76] CMS collaboration, Measurement of the ratio of the 3-jet to 2-jet cross sections in pp collisions at $\sqrt{s}=7 \mathrm{TeV}$, Phys. Lett. B 702 (2011) 336 [arXiv:1106.0647] [INSPIRE].

[77] PHENIX collaboration, A. Adare et al., Inclusive cross-section and double helicity asymmetry for $\pi^{0}$ production in $p+p$ collisions at $\sqrt{s}=200 \mathrm{GeV}$ : implications for the polarized gluon distribution in the proton, Phys. Rev. D 76 (2007) 051106 [arXiv: 0704.3599] [INSPIRE].

[78] PHENIX collaboration, A. Adare et al., Neutral pion production with respect to centrality and reaction plane in Au+Au collisions at $\sqrt{s_{N N}}=200 \mathrm{GeV}$, arXiv:1208.2254 [INSPIRE].

[79] R. Soltz et al., Constraining the initial temperature and shear viscosity in a hybrid hydrodynamic model of $\sqrt{s_{N N}}=200 \mathrm{GeV}$ Au+Au collisions using pion spectra, elliptic flow and femtoscopic radii, arXiv:1208.0897 [INSPIRE].

[80] M. Csanad and I. Majer, Equation of state and initial temperature of quark gluon plasma at RHIC, Central Eur. J. Phys. 10 (2012) 850 [arXiv:1101.1279] [InSPIRE].

[81] J.K. Nayak, J.-e. Alam, S. Sarkar and B. Sinha, Measuring initial temperature through photon to dilepton ratio in heavy ion collision, J. Phys. G 35 (2008) 104161 [arXiv: 0806.0447] [INSPIRE].

[82] M. Cacciari, J. Rojo, G.P. Salam and G. Soyez, Jet reconstruction in heavy ion collisions, Eur. Phys. J. C 71 (2011) 1539 [arXiv:1010.1759] [InSPIRE].

[83] M. Cacciari, G.P. Salam and G. Soyez, Fluctuations and asymmetric jet events in $\mathrm{PbPb}$ collisions at the LHC, Eur. Phys. J. C 71 (2011) 1692 [arXiv:1101.2878] [InSPIRE].

[84] G. de Barros, B. Fenton-Olsen, P. Jacobs and M. Ploskon, Data-driven analysis methods for the measurement of reconstructed jets in heavyion collisions at RHIC and LHC, arXiv: 1208.1518 [INSPIRE].

[85] A. Martin, W. Stirling, R. Thorne and G. Watt, Parton distributions for the LHC, Eur. Phys. J. C 63 (2009) 189 [arXiv:0901.0002] [INSPIRE].

[86] F. D'Eramo, M. Lekaveckas, H. Liu and K. Rajagopal, Momentum Broadening in Weakly Coupled quark-gluon Plasma (with a view to finding the quasiparticles within liquid quark-gluon plasma), arXiv:1211.1922 [INSPIRE]. 